\title{
Fixed-Wing Unmanned Aircraft In-Flight Pitch and Yaw Control Moment Sensing
}

\author{
Derrick Yeo,,- Ella M. Atkins,, \pm and Luis P. Bernal $\stackrel{ \pm}{ \pm}$ \\ University of Michigan, Ann Arbor, Michigan 48109 \\ and \\ Wei Shyy \\ The Hong Kong University of Science and Technology, Kowloon, Hong Kong, \\ People's Republic of China
}

DOI: $10.2514 / 1 . C 032682$

\begin{abstract}
Unsteady, nonlinear aerodynamics at high angles of attack challenges small unmanned aircraft system autopilots that rely heavily on inertial-based instrumentation. This work introduces an expanded aerodynamic sensing system for poststall flight conditions that incorporate high angle of attack and prop-wash aerodynamic forces based on in-flight measurement. A flight vehicle with a $1.8 \mathrm{~m}$ wingspan is used in wind-tunnel tests to measure the pitch and yaw moments due to freestream and prop-wash over the tail surfaces at high-thrust, low-airspeed conditions including hover. Test data are used to develop two methods to determine in-flight real-time pitch and yaw moments: a probe designed specifically to measure prop-wash flow and a set of pressure sensors embedded throughout the tail surfaces. Through comparisons with torque-transducer measurements also acquired in the wind-tunnel tests, both methods are shown to provide accurate moment estimates at hover and forward-flight conditions. With information directly provided by in-flight measurement, real-time pitch and yaw control can be enhanced using a simple and reliable framework.
\end{abstract}

\begin{tabular}{|c|c|}
\hline & Nomenclature \\
\hline$A_{M}, A_{N}$ & $=$ linear calibration factors \\
\hline$A R$ & $=$ wing aspect ratio \\
\hline$b$ & $=$ wingspan \\
\hline$C_{D}$ & $=$ aircraft drag coefficient \\
\hline$C_{L}$ & $=$ aircraft lift coefficient \\
\hline$C_{L_{\alpha}}$ & $=$ aircraft linear lift coefficient \\
\hline$C_{L_{0}}^{L_{\alpha}}$ & $=$ zero-alpha lift coefficient \\
\hline$C_{\mathcal{L}}, C_{\mathcal{L}_{\beta}}, C_{\mathcal{L}_{\delta_{a}}}, C_{\mathcal{L}_{\delta_{r}}}$ & $=$ roll moment coefficients \\
\hline 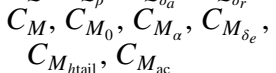 & $=$ pitch moment coefficients \\
\hline $\begin{array}{l}C_{N}, C_{N_{0}}, C_{N_{\beta}}, C_{N_{\delta_{a}}} \\
\quad C_{N_{\delta_{r}}}, C_{N_{\text {vtail }}}, C_{N_{\mathrm{ac}}}\end{array}$ & $=$ yaw moment coefficients \\
\hline$c$ & $=$ root chord, $\mathrm{m}$ \\
\hline$D$ & $=$ aircraft drag, $\mathrm{N}$ \\
\hline$e$ & $=$ Oswald efficiency factor \\
\hline$L$ & $=$ aircraft lift, $\mathrm{N}$ \\
\hline $\mathcal{L}$ & $=$ aircraft roll moment, $\mathrm{N} \cdot \mathrm{m}$ \\
\hline$\widetilde{L^{\prime}}$ & $=$ normal pressure force, $\mathrm{N}$ \\
\hline$l_{i}$ & $=$ tail surface panel moment arm, $\mathrm{m}$ \\
\hline$M$ & $=\quad$ aircraft pitch moment, $\mathrm{N} \cdot \mathrm{m}$ \\
\hline$N$ & $=$ aircraft yaw moment, $\mathrm{N} \cdot \mathrm{m}$ \\
\hline$n_{v / h \text { tail }}$ & $=$ number of tail surface panels \\
\hline
\end{tabular}

Presented as Paper 2013-4979 at the AIAA Atmospheric Flight Mechanics (AFM) Conference, Boston, MA, 19-22 August 2013; received 14 October 2013; revision received 1 March 2014; accepted for publication 8 March 2014; published online 28 January 2015. Copyright $@ 2014$ by Derrick W. Yeo, Ella M. Atkins, Luis P. Bernal, and Wei Shyy. Published by the American Institute of Aeronautics and Astronautics, Inc., with permission. Copies of this paper may be made for personal or internal use, on condition that the copier pay the $\$ 10.00$ per-copy fee to the Copyright Clearance Center, Inc., 222 Rosewood Drive, Danvers, MA 01923; include the code 1533-3868/15 and $\$ 10.00$ in correspondence with the CCC.

*Ph.D. Student, Aerospace Engineering Department. Student Member AIAA.

${ }^{\dagger}$ Associate Professor, Aerospace Engineering Department. Associate Fellow AIAA.

\#Associate Professor, Aerospace Engineering Department. Member AIAA.

\$Office of the Executive Vice-President and Provost, Mechanical Engineering Department, Room 6331, Academic Building (Lifts 13-15), Clear Water Bay; also Adjunct Professor, Aerospace Engineering Department, University of Michigan, Ann Arbor, MI 48109. Fellow AIAA.

$P$
$S$
$S_{h t a i l}$
$S_{v \mathrm{tail}}$
$V_{\mathrm{pw}}$
$V_{\mathrm{ac}}$
$\alpha$
$\alpha_{\mathrm{pw}}$
$\beta$
$\beta_{\mathrm{pw}}$
$\delta_{a}$
$\delta_{e}$
$\delta_{r}$
$\theta_{i}$
$\rho$

$=$ pressure $($ sensor data $)$

$=$ wing area, $\mathrm{m}^{2}$

$=$ horizontal tail surface area, $\mathrm{m}^{2}$

vertical tail surface area, $\mathrm{m}^{2}$

propeller-wash speed, $\mathrm{m} / \mathrm{s}$

aircraft airspeed, $\mathrm{m} / \mathrm{s}$

aircraft angle of attack, deg

propeller-wash probe pitch angle, deg

aircraft sideslip angle, deg

propeller-wash probe yaw angle, deg

aileron deflection angle, deg

elevator deflection angle, deg

rudder deflection angle, deg

panel deflection angle, deg

air density, $\mathrm{kg} / \mathrm{m}^{3}$

\section{Introduction}

$\mathbf{I}_{\mathrm{d}}^{\mathrm{N}}$ N THE last decade, unmanned aircraft systems (UASs) have been deployed for platform validation, science, and surveillance roles traditionally associated with manned aircraft $[1,2]$. Small fixed-wing UASs carrying modest payload weights are typically overpowered to the extent that even a single propeller-driven engine is capable of generating a thrust greater than total vehicle weight. This characteristic enables the small UAS to assume the advantages of fixedwing and rotary-wing platforms, specifically to cruise with the efficiency of a fixed-wing aircraft, to fly at high angles of attack and to hover over a site of interest or land/perch without the need for a runway.

The aerodynamics of high-angle-of-attack flight presents a number of challenges to fixed-wing aircraft. Near stall, aircraft lift coefficients are nonlinear with respect to parameters such as angle of attack and airspeed. Lateral handling issues such as asymmetric wing stall and wing rock also pose challenges at high angles of attack [35]. Early research focused on mitigating manned aircraft handling issues near stall and enhancing fighter jet maneuverability [5-8]. This body of knowledge remains largely applicable to engineers exploiting high-angle-of-attack flight on unmanned aircraft.

The current paradigm of small UAS instrumentation integrates inertial measurements supplemented by airspeed as a minimum or more capably a five-hole probe providing air-data measurements that include airspeed, angle of attack, and sideslip. Such systems have been successful in applications involving conventional fixed-wing flight within the traditional flight envelope [9-11]. Such platforms 
have provided a baseline capability for more advanced tests in areas such as cooperative control [12] and oceanborne operations [13]. Small autopilot systems such as the Kestrel Autopilot [14] and Micropilot are also capable of serving fixed- and rotary-wing vehicles [15]. However, high-angle-of-attack fixed-wing aerodynamics challenges traditional UAS autopilot instrumentation and control laws, which typically rely on a single linear relationship between angle of attack and coefficient of lift. Flight near or beyond stall causes flow separation that results in aerodynamics that are unsteady, nonlinear, and sensitive to small changes in flight conditions and cannot be handled by linear controllers $[\underline{16}, 17]$ with a traditional instrumentation scheme.

Working within the limitations of the current small UAS instrumentation framework, a number of fixed-wing UAS have been guided between cruise and hover in the last decade. Green and Oh developed indoor hobby aircraft that could autonomously transition from cruise to hover using inertial measurements and a linearized controller [18]. This autonomous fixed-wing hovering demonstration did not model the high-angle-of-attack flight regime; instead, it used airframe properties such as low rotational inertia, high thrust/weight ratios, and a control law that allowed it to "bully its way through stall." A similar approach in avoiding the aerodynamic problems at high angles of attack was taken by Frank et al. [19], achieving successful autonomous transitions to hover and docking in a Vicon motion-capture environment. More recently, Cory and Tedrake [20] used the Vicon system to provide valuable insight into the nonlinear aerodynamic effects encountered during a perching maneuver. Their work represents a step toward exploiting the aerodynamic phenomena at stall instead of avoiding it. An aerodynamic coefficient estimator based on angle of attack and elevator deflection given still indoor air and the kinematic data from the Vicon environment was formulated based on their findings. Selig [21,22] developed a component-based approach to modeling the aerodynamics of highthrust radio-controlled (RC) aircraft flying aerobatic maneuvers, noting that strong nonlinearities and coupling effects challenge a traditional stability derivative approach.

Johnson and Kannan [23] developed an adaptive controller that enabled autonomous transitions to and from hover. The guidance law used during the transition was similar in formulation to those used in previous work; the commanded inertial pitch angle was set to vertical to achieve the transition. A slow ramp approach and a faster stepchange transition were tested, and it was noted that both resulted in significant altitude tracking error during the transition. Johnson and Kannan suggested that an airspeed-bleed strategy could be a potential solution.

In the authors' previous work [24], the use of pressure-based aerodynamic sensing to support such a transition guidance phase was proposed. Processing pressure data over an instrumented wing chord, the autopilot was able to detect stall and reliably bleed airspeed up to the point when stall was detected before switching control modes. Flight tests [25] showed that expanded aerodynamic data could improve the ability of a simple, linear decoupled autopilot to operate under nonlinear conditions. This paper seeks to develop the concept of expanded aerodynamic data for small UAS by using an expanded set of pressure measurements across the aircraft for additional flow information.

The concept of pressure-based estimation of the flowfield above an airfoil has been a cornerstone of wind-tunnel testing, but in stepping toward aerodynamics-based feedback, the most relevant efforts to this work have focused on enabling closed loop feedback in "onboard" active flow control schemes to alleviate flow separation or emulate control surfaces through the use of flow actuation. One successful example was implemented by Patel et al. [26,27], who considered the time-domain response from a high-bandwidth pressure sensor to predict incipient flow separation at the wing leading edge and trigger the activation of a plasma flow actuator. Under attached flow conditions, Cox et al. [28] used pressure-based estimates of the lift curve above an airfoil as feedback for an automated cruise flap. NASA has supported wind-tunnel-based implementation and testing of a distributed actuation and sensing array for use on a blended wing body UAS, using a series of pressure measurements to study the effectiveness of a morphing wing control strategy [29,30]. The U.S. Air Force Office of Scientific Research Adaptive VOrticity Control Enabled flighT (AVOCET) project [31] aims to continuously tailor the pressure distribution and resulting forces and moments across the wing using advanced microtuft sensors and hybrid fluidic flow actuators.

The overall goals of the aerodynamic feedback concept proposed in this research bridges the two efforts described previously. The existing active flow control framework developed by Patel et al. [26] and Bowles and Corke [27] is suited to alleviate retreating blade stall in rotorcraft and expand their performance envelope. With the AVOCET project, flow measurement and actuation across the wing surfaces are closely coupled to vehicle control and the system aims to achieve a careful tailoring of vorticity distributions in real time to also alleviate gusts and flow disturbances. Our objective is to show that the proposed aerodynamic sensing system can provide means to extend the envelope of current small UAS autopilots through use of the proposed modeling scheme plus real-time pressure sensor feedback.

It should be noted that, although the intended purpose of the aerodynamic sensing presented in this work is to offer additional data for feedback control, our objectives are not to directly affect the flow structure but to provide improvements on how the conventional surfaces can be used. A series of wind-tunnel tests are conducted to examine the capabilities of the instrumentation package and to provide real-time control surface aerodynamic moment estimates in a controlled test environment. Of particular interest for slow and hovering flight is the ability to directly measure and model pitch and yaw moments generated by the elevator and rudder in poststall conditions when the freestream flow is insufficient to produce significant stabilizing and maneuvering moments with the tail surfaces, but where prop-wash provides this flow. The experimental approach presented next uses a full-scale UAS platform in a $5 \times 7 \mathrm{ft}$ wind-tunnel test section. Wind-tunnel tests conducted previously on full-scale small UAS have characterized aerodynamic characteristics of an aircraft [32] for novel control strategies [33,34]. Using the actual flight vehicle as a test model allows for testing when the propulsion system is active. Landman et al. investigated the longitudinal and lateral aerodynamic characteristics of a small UAS with and without power applied to the propeller [35]. Recent work by $\mathrm{Ol}$ et al. characterized the low-airspeed high-thrust aerodynamics of an aerobatic RC airframe in the presence of prop-wash using transducer-based instrumentation and found that high thrust settings at low advance ratios serve to linearize control-surface response [36].

This paper seeks a novel approach to fixed-wing UAS instrumentation that meets the challenges of high-alpha flight through expanded sensing and reformulation of the steady-flight equations to incorporate poststall aerodynamic force based on in-flight measurement. Consequently, real-time pitch and yaw control can be enhanced using a simple and reliable framework.

\section{Steady Level Flight Equations}

The steady-flight equations represent the most basic principles of flight mechanics [37] and are used as the foundation for the development of small UAS autopilots. In this section, the aerodynamic force and moment equations for an aircraft in steady flight are described. Under the steady-flight assumptions, the forces generated by the main wing are used to characterize the lift and drag generated by the aircraft. These are given by the aerodynamic equations for lift and drag:

$$
\begin{aligned}
& L=\frac{1}{2} \rho V_{\mathrm{ac}}^{2} S C_{L} \\
& D=\frac{1}{2} \rho V_{\mathrm{ac}}^{2} S C_{D}
\end{aligned}
$$

where $\rho$ is the atmospheric density, and $V_{\text {ac }}$ is the freestream airspeed of the aircraft. The lift and drag coefficients $C_{L}$ and $C_{D}$ depend linearly on the aircraft angle of attack $\alpha$. This linear relationship is 
a good model up to the point of wing stall and flow detachment across the lifting surfaces:

$$
\begin{gathered}
C_{L}=C_{L_{0}}+C_{L_{\alpha}} \alpha \\
C_{D}=C_{D_{0}}+\frac{C_{L}^{2}}{\pi e A R}
\end{gathered}
$$

The lift coefficient is the combination of a constant offset $C_{L_{0}}$ at zero angle of attack, and the linear lift coefficient associated with the wing $\left(C_{L_{\alpha}}\right)$. The drag coefficient similarly features a constant parasite drag constant $C_{D_{0}}$ and a term that is dependent on lift, the aspect ratio $(A R)$ of the wing, and the Oswald efficiency factor $e$ associated with its planform. Under steady-flight assumptions, the pitch moment equation for an aircraft is

$$
M=\frac{1}{2} \rho V_{\mathrm{ac}}^{2} S c C_{M}
$$

where $c$ is the root chord of the wing by convention, and $S$ is the surface area of the wing. The previous steady-flight equations inherently assume that the incoming wind vector can fully represent the longitudinal aerodynamic forces and moment of the entire aircraft. The nondimensional pitch moment coefficient $C_{M}$ combines the effects of all aerodynamic surfaces such as the wings, fuselage, and horizontal stabilizer. It is a linear function of the aircraft angle of attack $\alpha$ and elevator deflection $\delta_{e}$ as given in

$$
C_{M}=C_{M_{0}}+C_{M_{\alpha}} \alpha+C_{M_{\delta_{e}}} \delta_{e}
$$

where the coefficients $C_{M_{0}}, C_{M_{\alpha}}$, and $C_{M_{\delta_{e}}}$ are determined through theoretical models or wind-tunnel testing. Under steady-flight conditions where neither the aircraft nor the horizontal tail is stalled, this linear relationship of predetermined coefficients and the aircraft wind vector can be used to approximate aircraft pitch moments.

The lateral forces and moments, side force, yaw moment, and roll moment must also be balanced for steady flight. For steady flight, aerodynamic side force application is typically not appreciable, although sideslip can be used to reduce energy on approach, for example. In this work, focus is placed on use of the prop-wash as a means of controlling the aircraft in poststall conditions. Presuming sufficient flow over the tail, the vertical stabilizer and rudder can use the prop-wash to control yaw, as will be explored in this paper. Because the horizontal and vertical tail sections are completely immersed in the prop-wash, they are considered to be more relevant to the current research. This paper therefore focuses on longitudinal pitching moment and lateral yawing moment, which can be controlled by the tail surfaces. Below the equations for yaw and roll moment are presented. The equation for yaw moment $N$ is

$$
N=\frac{1}{2} \rho V_{\mathrm{ac}}^{2} S b C_{N}
$$

where $b$ is the wingspan. The yaw moment coefficient $C_{N}$ is a linear function of the aircraft sideslip angle, aileron deflection $\delta_{a}$, and rudder deflection $\delta_{r}$, as given in

$$
C_{N_{\text {combined }}}=C_{N_{\beta}} \beta+C_{N_{\delta_{a}}} \delta_{a}+C_{N_{\delta_{r}}} \delta_{r}
$$

where $C_{N_{\delta_{0}}}$ is the yaw moment due to the differential drag caused by aileron deflection. The aerodynamic roll moment is given by

$$
\mathcal{L}=\frac{1}{2} \rho V_{\text {ac }}^{2} S b C_{\mathcal{L}}
$$

where the roll coefficient depends linearly on slip angle $\beta$, aileron deflection $\delta_{a}$, and rudder deflection $\delta_{r}$ :

$$
C_{\mathcal{L}_{\text {combined }}}=C_{\mathcal{L}_{\beta}} \beta+C_{\mathcal{L}_{\delta_{a}}} \delta_{a}+C_{\mathcal{L}_{\delta_{r}}} \delta_{r}
$$

In summary, the steady-flight force and moment equations based on relative wind velocity vector and a series of predetermined coefficients enable a straightforward computation of aerodynamic forces and moments across the vehicle. Although suitable for most forward-flight conditions, this formulation is not applicable during operations beyond stall when the aircraft wind vector may not represent the local flow over the flight surfaces. A reformulation of these aerodynamic moment equations is proposed below that includes additional components representing prop-wash as well as freestream velocity terms.

During operations outside the envelope of conventional forward flight such as at high angles of attack, effects such as flight surface stall and unsteady flowfields across the vehicle mean that simple wind vector measurements and predetermined coefficients will be incapable of accurately modeling the aerodynamic forces and moments generated by an aircraft. However, unsteady but periodic flow introduced by phenomena such as prop-wash creates a quasisteady flow condition that can still be modeled by an extended set of equations that account for prop-wash as well as freestream flow.

A simplification of the basic lift and drag equations are proposed based on direct measurements taken over the surface of the wing, where the measured force normal to the chord line of left and right wings are $L_{\text {Left }}^{\prime}$ and $L_{\text {Right }}^{\prime}$, respectively:

$$
\begin{gathered}
L=\left(L_{\text {Left }}^{\prime}+L_{\text {Right }}^{\prime}\right) \cos \alpha \\
D=\frac{1}{2} \rho V_{\text {ac }}^{2} S C_{D_{0}}+\left(L_{\text {Left }}^{\prime}+L_{\text {Right }}^{\prime}\right) \sin \alpha
\end{gathered}
$$

The aerodynamic lift is computed as the component of the combined pressure force from both wings that is normal to the wind vector. The drag is the combination of the parasitic drag coefficient $C_{D_{0}}$ multiplied by dynamic pressure and the component of the main wing pressure force in the direction of the wind vector. The parasitic drag coefficient can be determined through wind-tunnel testing.

Consider a propeller-driven, fixed-wing aircraft with a conventional configuration but sufficient thrust to at least balance (lift) the full aircraft weight. Such an aircraft certainly can maintain altitude or potentially even climb without relying on wing-generated lift, but the wings and tail will still provide aerodynamic forces and moments that can be used to stabilize and maneuver the aircraft. To sustain flight at high angles of attack beyond stall, the aircraft must rely more heavily on propeller thrust to balance its weight, resulting in high-speed propwash causing local flow regions that differ greatly from the vehicle wind vector. Further, the interaction between the aircraft wind vector and the wind vector at high angles of attack make a mapping between throttle setting and the propeller prop-wash challenging.

This work proposes the definition of a new prop-wash velocity variable $V_{\mathrm{pw}}$ and separation of the moment coefficient terms for the freestream versus prop-wash velocities to better account for differing flow conditions at quasi-steady high-alpha or hover-flight conditions. The equations presented in this section propose an expansion on the steady level-flight equations to accommodate additional sensing and provide moment estimates that remain valid at high angles of attack and hover. First consider the pitch moment equation. With additional prop-wash-induced velocity $V_{\mathrm{pw}}$ over the tail surfaces, the equation can be rewritten using separate aerodynamic coefficients for the horizontal tail $C_{M_{h t a i l}}$ and the fuselage/wing combination $C_{M_{\mathrm{ac}}}$ as

$$
\begin{gathered}
M=\frac{1}{2} \rho V_{\mathrm{ac}}^{2} S c C_{M_{\mathrm{ac}}}+\frac{1}{2} \rho V_{\mathrm{pw}}^{2} S_{h \text { tail }} l_{\text {tail }} C_{M_{h \text { tail }}} \\
C_{M_{\mathrm{ac}}}=C_{M_{\mathrm{ac}_{0}}}+C_{M_{\mathrm{ac}_{\alpha}}} \alpha \\
C_{M_{h \text { tail }}}=C_{M_{h \text { tail }_{0}}}+C_{M_{h \text { tail } \alpha}} \alpha_{\text {tail }}+C_{M_{h \text { tail }_{\delta_{e}}}} \delta_{e}
\end{gathered}
$$

The coefficients $C_{M_{h \text { tail }_{0}}}, C_{M_{\text {tail }_{\alpha}}}$, and $C_{M_{h \text { tail }_{\delta_{\rho}}}}$ describe the pitch characteristics of the horizontal tail surfaces and can be determined through wind-tunnel testing. Under prestall steady-flight conditions, both velocities will be comparable, and this formulation 
approximates the conventional pitch moment equation. However, during low-speed high-angle-of-attack flight, the prop-washinduced velocity begins to dominate the flowfield. The proposed reformulation including prop-wash flow allows the pitch moments generated by the elevator to be estimated past freestream stall and in hover. The second term, $\frac{1}{2} \rho V_{\mathrm{pw}}^{2} S_{h \text { tail }} l_{\text {tail }} C_{M_{h \text { tail }}}$, can be measured using distributed pressure sensing, or using additional inflow information such as provided by our custom prop-wash probe discussed next. The reformulation for the yaw moment equation follows in a similar manner for the vertical tail, as shown next:

$$
\begin{gathered}
N=\frac{1}{2} \rho V_{\mathrm{ac}}^{2} S b C_{N_{\mathrm{ac}}}+\frac{1}{2} \rho V_{\mathrm{pw}}^{2} S_{v \text { tail }} l_{]} C_{N_{v t a i l}} \\
C_{N_{\mathrm{ac}}}=C_{N_{\mathrm{ac}_{0}}}+C_{N_{\mathrm{ac}_{\beta}}} \beta \\
C_{N_{\text {vtail }}}=C_{N_{\text {vtail }_{0}}}+C_{N_{\text {vtail }_{\beta}}} \beta_{\text {tail }}+C_{N_{\text {vtail }_{\delta_{r}}}} \delta_{r}
\end{gathered}
$$

As with the pitch moment equation, the second term can be estimated through direct aerodynamic measurements or through predetermined coefficients in conjunction with a velocity measurement as shown previously. The coefficients $C_{N_{v_{\text {tail }}}}, C_{N_{v_{\text {tail }}}}$, and $C_{N_{v_{\text {tail }} \delta_{\delta_{r}}}}$ can be determined through wind-tunnel testing.

The moments associated with the tail surfaces are amenable to "partial" and "complete" sensing strategies. Because the prop-washinduced velocities are large and the tail surfaces may operate in an attached flow condition even when in a hover, it could be sufficient to use an additional velocity measurement in conjunction with a new set of coefficients. With a complete distributed pressure sensing strategy, the entire moment contribution over the tail surfaces can be measured directly. If the prop-wash-induced flow speeds are low or the control surface deflections are too large to ensure attached flow over the tail surfaces, the distributed pressure measurement strategy over the tail can still provide accurate estimates of yaw and pitch moments.

For roll, an approach that uses direct measurements is necessary. When the main wings operate in a poststall, separated flow regime, the aerodynamics are nonlinear and unsteady. Further, the prop-wash over the wings may provide significant control authority to the ailerons at high-angle-of-attack flight. Although a study of the wingin-slipstream effects is beyond the scope of this paper, the roll moment equation presented next uses estimates of differential lift over the wings in conjunction with additional logic for aileron authority:

$$
\begin{gathered}
\mathcal{L}=\frac{1}{2} \rho V_{\text {ac }}^{2} S b C_{\mathcal{L}_{\beta}} \beta+\frac{1}{2} \rho V_{\text {pw }}^{2} S_{v \text { tail }} l_{\text {tail }} C_{\mathcal{L}_{\delta_{r}}} \delta_{r}+\mathcal{L}_{\text {wings }} \\
\mathcal{L}_{\text {wings }}=\left(L_{\text {Left }}^{\prime}-L_{\text {Right }}^{\prime}\right) b_{\text {eff }} \\
L_{\text {Left }}^{\prime}=f\left(\delta_{a_{\text {Left }}}\right) \\
L_{\text {Right }}^{\prime}=f\left(\delta_{a_{\text {Right }}}\right)
\end{gathered}
$$

Here, $L_{\text {Left }}^{\prime}$ and $L_{\text {Right }}^{\prime}$ are direct measurements of the aerodynamic forces normal to the wing surface. The effective roll moment $\operatorname{arm} b_{\text {eff }}$ can either be determined based on the geometry of the wing or determined through wind-tunnel testing, depending on the particular lift sensing scheme used. Tests and results described in the remainder of this paper focus on the pitch and yaw sensing aspect of the proposed steady-flight equation reformulation, which are the two values most directly influenced by propeller-induced flow.

\section{Embedded Aerodynamic Sensing System}

The instrumentation package proposed in this work is based on a set of pressure measurements taken across the aircraft through pressure ports and through multihole probes. An overview of the sensing system is shown in Fig. 1. At each indicated location,

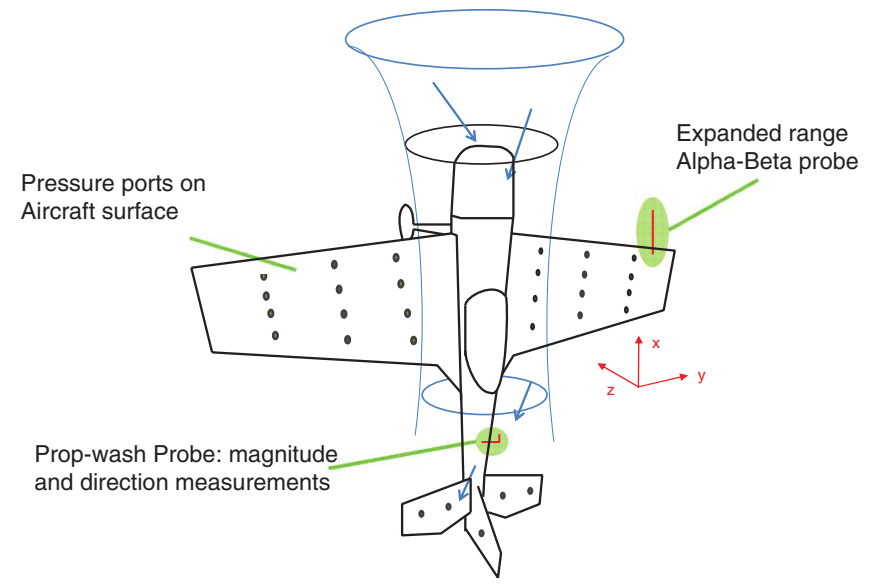

Fig. 1 Embedded aerodynamic sensing system.

pressure ports on the top and bottom surface are connected to a locally mounted differential pressure sensor.

Although the Fig. 1 schematic also shows distributed sensing in the wing, this paper focuses on carefully establishing moments associated with prop-wash flow over the tail. Details of the wing instrumentation are given in an earlier publication [38]. Thus, while the instrumentation design is proposed for all lift surfaces, results in this paper focus on data acquired from tail surfaces only.

\section{A. Embedded Pressure Sensors on Flight Surfaces}

At high angles of attack, the flow across the tail surfaces can differ greatly from the flow conditions encountered by the aircraft in general. This is due to the incident flow caused by the high-velocity propeller wash, which is not typically considered by UAS autopilots. The aerodynamic sensing strategy for the flight vehicle tail section was conceived to provide flowfield information across the tail surface during uncertain flight conditions and allow real-time estimates of control surface authority. Using differential pressures comparing top and bottom pressure at the same surface station as well as the area integration scheme, lift estimates can be calculated. The schematic in Fig. 2 depicts the flight vehicle tail surfaces and provides an overview of the geometry used to discretize the tail surfaces and perform the surface pressure integration.

The pressure ports are connected to Honeywell HSCDR 1NDAA5 pressure sensors with $\mathrm{a} \pm 1$ inch of $\mathrm{H}_{2} \mathrm{O}\left(\mathrm{in}_{2} \mathrm{O}\right)$ measurement range and a $0.5 \mathrm{~ms}$ response time. These are the most sensitive sensors in the HSCDR series that are small enough to be located within the surfaces, allowing for short and uniform pressure line lengths of approximately $40 \mathrm{~mm}$ between ports and sensors. An experimental characterization was carried out using an acoustic driver that caused pressure excitations at fixed frequencies. A pressure transducer was connected via different lengths of tubing and the signal compared to a reference transducer that was connected directly to the driver. This characterization was used to verify that differences in response between 1 and $40 \mathrm{~mm}$ lengths of tubing was not measurable at frequencies between 50 and $150 \mathrm{~Hz}$. To accommodate the instrumentation, additional structure was fabricated and built into the tail surfaces. The ports and sensors are connected to provide differential pressure between top/bottom and left/right surfaces. The differential pressure $P_{\text {diff- } i}$ is measured across the horizontal tail, elevators, vertical tail, and rudder at $i$ locations. Each location corresponds to a panel of area $S_{h \text { tail } i}$ that is $l_{i}$ from the center of gravity and has a deflection angle of $\theta_{i}$. This deflection angle is zero for panels on the stabilizers and equal to the control surface deflection angle for panels on the rudder and elevator. As such, the terms relating to the control surfaces can be rewritten as follows.

Measured horizontal tail pitch moment contribution:

$$
\sum_{i=1}^{n_{\text {htail }}} \cos \theta_{i} \cdot P_{\mathrm{diff}-i} \cdot S_{h \text { tail } i} \cdot l_{i}
$$




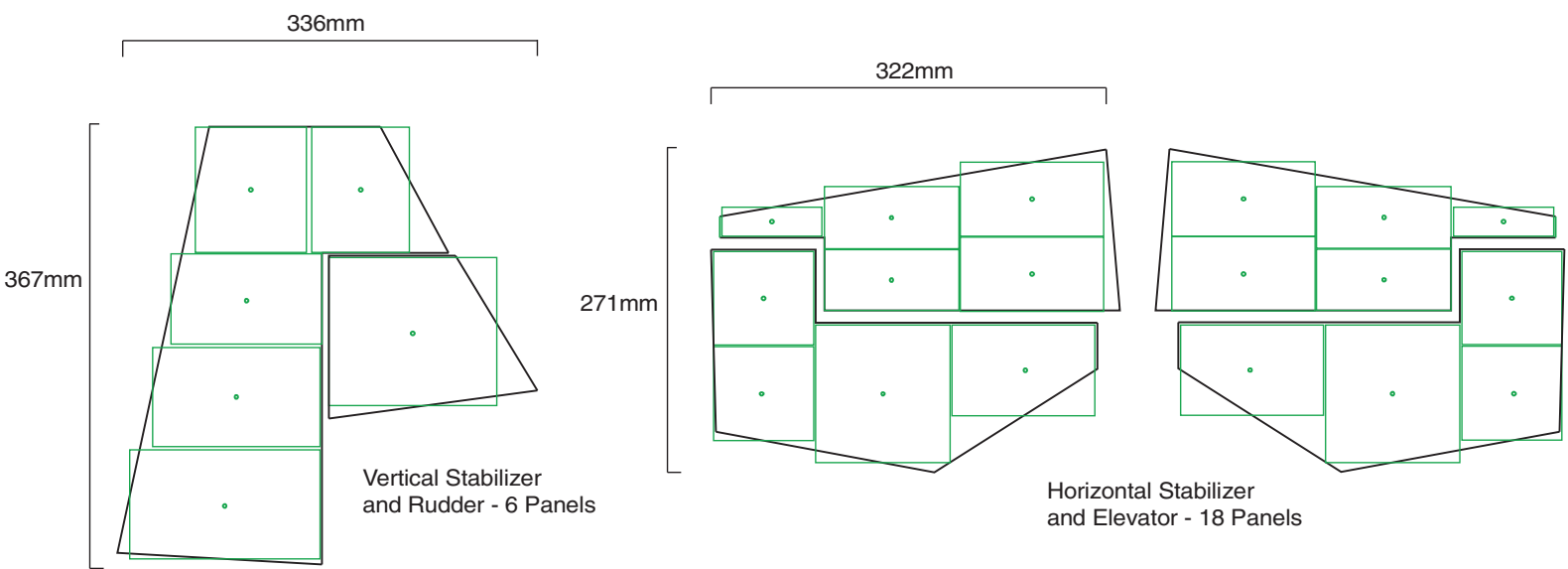

Fig. 2 Distributed sensing over tail surface and area discretization scheme.
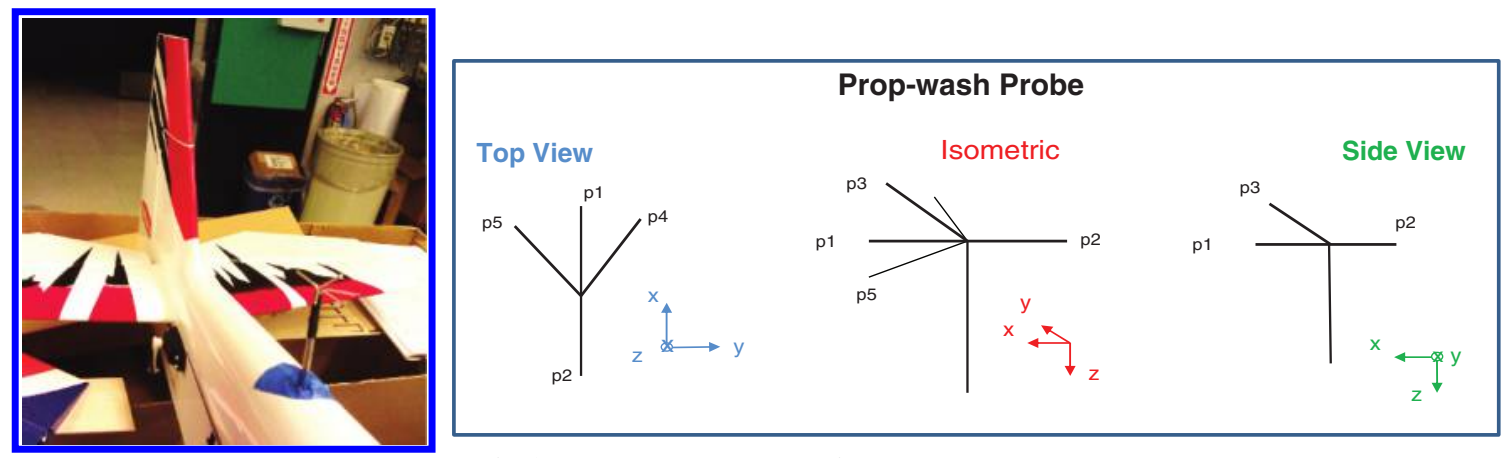

Fig. 3 Prop-wash probe location and geometry.

Measured horizontal tail pitch moment contribution:

$$
\sum_{i=1}^{n_{v t \text { tail }}} \cos \theta_{i} \cdot P_{\mathrm{diff}-i} \cdot S_{v \text { tail } i} \cdot l_{i}
$$

\section{B. Dedicated Multihole Probe for Prop-Wash Estimation}

Key assumption of current fixed-wing autopilot systems is that aerodynamic flight control authority is strictly a function of the freestream wind vector. Because the flow speed over control surfaces typically scales according to flight speed, the ability to generate aerodynamic forces and moments using control strategies such as gain scheduling are commonly tied to freestream airspeed and angle of attack. Although this is reasonable for most fixed-wing forwardflight regimes, it is not accurate during aggressive maneuvering or operations past stall.

For a fixed-wing aircraft with excess thrust such as the present flight vehicle, during low-speed flight and hover the thrust generated by the propeller plays a significant role in supporting the aircraft in flight. Further, the single-propeller puller configuration induces significant airflow across the tail control surfaces at moderate to high throttle settings. In hover, the effectiveness of the control surfaces to generate aerodynamic force and moments depend solely on the propwash, which itself creates moments of its own due to the swirl imparted by the blades.

To this end, a custom-designed multihole probe was built to measure prop-wash velocity and angle of incidence on the tail surfaces. It was designed to measure flow speed and inflow angles in two axes by using a set of prongs, with each terminating in a small pressure port. Its operating principle is similar to a cobra probe [39], but one key difference is that it uses an aft-facing pressure port for a local estimate of dynamic pressure as well as an ability to characterize reverse flow in aerobatic conditions such as "backsliding" at zero thrust. The probe is mounted in front of the tail section and located approximately one vertical stabilizer chord length upstream to avoid excessive interference with the flow over the vertical tail. The geometry of the probe and a picture of it mounted on the flight vehicle fuselage are shown in Fig. 3.

The probe is built using aluminum tubing and provides flow angle and speed measurements by comparing the pressure measured across its prongs and is configured to use three differential pressure sensors. The current sensor configuration was chosen based on the expected pressure magnitudes encountered during wind-tunnel testing and is summarized in Table 1. A negative pressure indicates a negative pressure difference between the indicated ports. The procedure used to calibrate the prop-wash probe is described in [표].

\section{Wind-Tunnel Test Model Configuration}

The Hangar-9 Funtana X100/X125 is the commercial off-the-shelf small UAS flight vehicle chosen for the wind-tunnel experiments. It has a wingspan of $1.8 \mathrm{~m}$ and a maximum weight of $5 \mathrm{~kg}$. In addition to being fully aerobatic, it also supports an appreciable avionics payload while maintaining sufficient thrust to stably hover, specifically a thrust-to-weight ratio larger than 1.5 when fully loaded with an avionics payload of $660 \mathrm{~g}$. A Funtana X125 fuselage was developed as a wind-tunnel test model for ground-based testing. It retains all the systems necessary for RC flight and includes additional internal structure for mounting a force-torque sensing system. An overview of the complete platform is shown in Fig. 4 . The key requirements of the test platform are a suitable propulsion system, a force-torque sensor, and the relevant components of the aerodynamic sensing system.

Table 1 Prop-wash probe sensor configuration and port assignment

\begin{tabular}{lccc}
\hline \hline $\begin{array}{l}\text { Probe } \\
\text { measurement }\end{array}$ & Pressure sensor & $\begin{array}{c}\text { Sensor range, } \\
\text { in } \mathrm{H}_{2} \mathrm{O}\end{array}$ & $\begin{array}{c}\text { Pressure port } \\
\text { connection }\end{array}$ \\
\hline$V_{\mathrm{pw}}$ & HSCDR 2NGAA5 & -0.3 to 2 & P1-P2 \\
$\alpha_{\mathrm{pw}}$ & HSCDR 1NDAA5 & -1 to 1 & P1-P3 \\
$\beta_{p w}$ & HSCDR 1NDAA5 & -1 to 1 & P5-P4 \\
\hline \hline
\end{tabular}




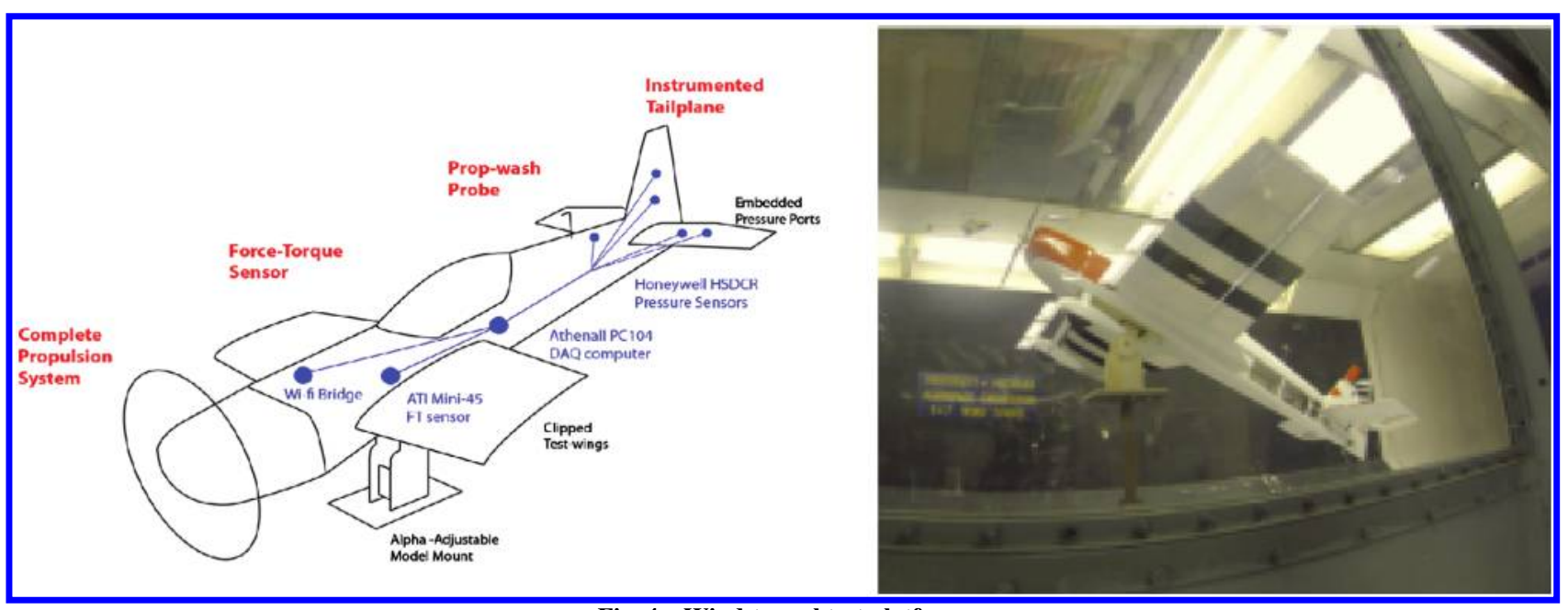

Fig. 4 Wind-tunnel test platform.

For these tests, primary sensors include a fuselage mounted propwash $(\mathrm{PW})$ probe and instrumented tail section, described in more detail next.

A Diamond Systems 1 Athena II PC104 computer is used for 16 bit analog data acquisition at $1 \mathrm{kHz}$. The Athena is configured to communicate with an external laptop through a wireless access point. The ATI Industrial Automation-* Mini-45 force-torque sensor has a maximum load capacity of $145 \mathrm{~N}$ in the $x$ and $y$ directions and $290 \mathrm{~N}$ in the $z$ direction. It is mounted internally with the tool tip facing outward. This allows the test model to be fully self-contained with no external wiring. The model is mounted on a custom-built, two-part stand that is adjustable for angle of attack and features a quick-release bolt system to allow the model to be quickly repositioned. More details on the probe calibration process can be found in previous work [38].

\section{Experimental Procedure}

A series of wind-tunnel tests were designed to evaluate the aerodynamic data system and to characterize prop-wash-induced aerodynamic moments of the test platform under controlled and repeatable quasi-steady flow conditions. The flow across the tail surfaces was changed by varying wind-tunnel freestream velocity, propeller revolutions per minute, and model mounting angle in the wind tunnel. As can be seen in Fig. 4, flow blockage in the windtunnel test section is significant and affects the ability of the experimental configuration to accurately recreate flight conditions outside of a steady hover. This is not considered a limitation to the current work because this paper focuses on the pressure-based instrumentation to provide onboard measurements of existing moments rather than the determination of specific airframe characteristics. A series of tail control surface deflection sweeps were carried out over a range of flow conditions. These include an elevator sweep with the rudder at zero deflection, a rudder sweep with the elevator at zero deflection, and a coarse combined rudder/elevator deflection sweep. The deflection ranges and step size were varied for each test condition, depending on the maximum moments generated by the control surfaces to accommodate saturation limits of the embed4ded ATI force-torque sensor. An overview of this test series is shown in Fig. 5 .

At a given propeller revolutions per minute, elevator and rudder deflection sweeps were carried out as described previously. These were repeated for model mounting angles of positive 25,0 , and negative $25 \mathrm{deg}$. When mounted at a nonzero angle of attack, the tail of the aircraft was close to the test section floor due to the limited test section dimensions. The tunnel structure was thus expected to interact with the prop-wash, resulting in modified inflow conditions

IData available online at http://www.diamondsystems.com [retrieved 23 July 2014].

**Data available online at http://www.ati-ia.com [retrieved 23 July 2014].

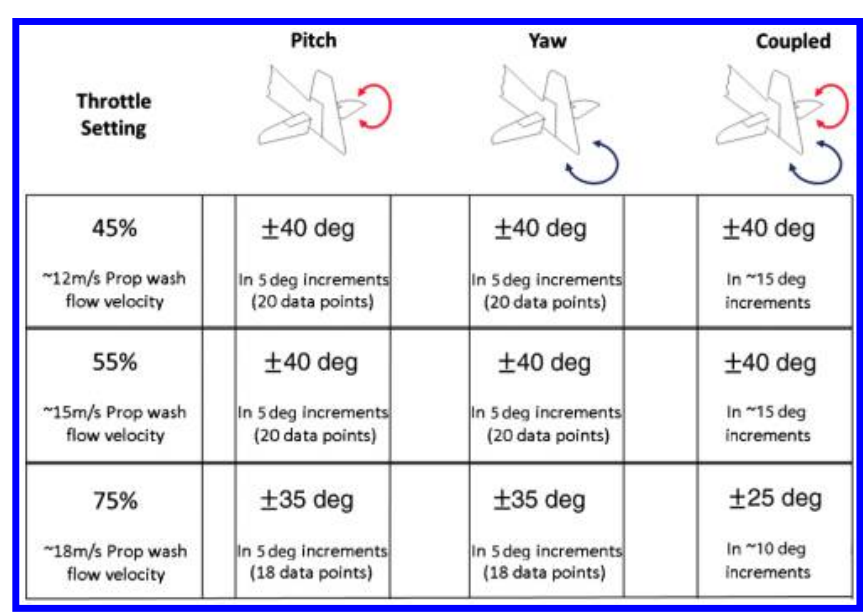

Fig. 5 Basic test procedure overview; tests performed at $\alpha=25$, 0 , and -25 deg.

on the tail. Because the test was intended to compare different sets of instrumentation instead of specifically characterizing the Funtana aircraft, this was not considered a prohibitive limitation for the current work. Data were also collected with the propeller fixed (no thrust) and a wind-tunnel freestream of $12 \mathrm{~m} / \mathrm{s}$. In this case, all flow is driven by the low-turbulence freestream, allowing the instrumentation to be evaluated without propeller wash.

\section{E. Data Processing and Analysis}

To compare aerodynamic pressure and ATI force-torque (FT) sensor measurements of tail-section control moments, the raw $1 \mathrm{kHz}$ data from both sets of instrumentation must be preprocessed appropriately. The force-torque sensor readings were time averaged over the sample window and the mean and standard deviation determined. Because of the turbulent nature of the flow behind a propeller, a filtering scheme was developed to deal with the additional signal noise.

The raw pressure data are subject to significant noise that manifests itself as fluctuations about the average value that is representative of a given test condition. This can be quantified through the standard deviation of each data set, and the raw measurements show standard deviation intervals that span nearly $20 \%$ of the full measurement range. Although a postprocessing filter methodology is suitable in an experimental scenario, a flight-ready filter algorithm provides a more relevant estimate of how the aerodynamic instrumentation will perform when in the air. This section documents the approach taken to filtering raw pressure data collected during Funtana windtunnel tests. 
To compile average measurements over the expected turbulent propeller flow conditions, a simple moving average filter (SMA) was chosen. An $n$-point SMA is computed as the unweighted mean of the previous $n$ measurements. For a series of $n$ measurements $P_{M}, P_{M-1}, \ldots P_{M-(n-1)}$, the SMA at $M$ is

$$
\mathrm{SMA}=\frac{P_{M}+P_{M-1}+, \ldots P_{M-(n-1)}}{n}
$$

where the period is the sampling rate multiplied by the number of averaged points $n$ and may be chosen based on the fluctuations present in the data. A period that is too short in comparison to the time scale of the main disturbance will result in excessive noise in the final signal, while a period that is too long will cause the aerodynamic data system to react too slowly to changing flight conditions. The following subsections describe the methodology used in determining a suitable period for the SMA filter.

For operations within the wake of a propeller, it is assumed that the most important disturbance time scale will depend on propeller rotation rate. To verify this, a survey of SMA periods for different test cases was conducted. The standard deviation of each data set about an average value was chosen as a metric of filter performance. At each test revolutions per minute, a control surface sweep is performed, and time averages at each elevator deflection are computed along with the standard deviation. The average width of the standard deviation for all deflection angles in the sweep is computed and normalized by the maximum pitch moment encountered. This normalized value represents the overall standard deviation for each revolutions per minute and is computed using a different number of SMA points. In developing the filtering parameters, data from elevator sweeps are used.

A normalized SMA period was established based on the two-perrevolution tip vortex excitation caused by each of the two propeller blades. This is based on the simplified assumption that the wake shed by each rotating blade is the main cause of pressure fluctuations in the data. This gives the SMA period for a particular test revolutions per minute as

$$
T_{\mathrm{SMA}}=\frac{1}{2} N_{\mathrm{wakes}} \cdot \frac{60}{\mathrm{RPM}}
$$

where $T_{\mathrm{SMA}}$ refers to the time taken for approximately $N_{\text {wakes }}$ tip vortices to be convected past the tail section. Because revolutionsper-minute measurements may not always be available to the aerodynamic data system, this filtering strategy can be reformulated to use prop-wash velocity measurements and known characteristics of the propeller. Blade pitch is a unit of length defined as the distance advanced by the propeller over one full revolution if it turned in a solid medium with no slippage. It is customary to use the pitch angle of the blade at a radial point that is $70 \%$ of blade diameter [40]. As such, a frequency that is analogous to propeller revolutions per minute can be obtained by dividing the measured prop-wash velocity by the pitch of the propeller. More details on the calibration process can be found in previous work [38]:

$$
T_{\mathrm{SMA}_{\text {velocity }}}=\frac{1}{2} N_{\text {wakes }} \cdot \frac{\text { Propellor Pitch }}{V_{\mathrm{pw}}}
$$

\section{Test Results: Propeller-Dominated Flow}

Hover test results are presented next, followed by low-airspeed and powered-cruise case studies. Test data will be summarized in a series of plots that describe the moments measured using both pressure $P$ and force-torque (FT) instrumentation with changing control-surface deflections. The data are presented in natural units of torque because the vehicle airspeed at the studied flight conditions is not suited to a coefficient-based approach. Collected data are used to compute coefficients in the steady-flight equations reformulated for highangle-of-attack and propeller-driven hovering flight.

\section{A. Hover Test Results}

This section investigates the use of the distributed pressure sensing to better capture and model yaw and pitching moments induced by prop-wash during hover. For this series of experiments, a simulated hover condition was established in the wind tunnel by mounting the vehicle level and leaving the wind-tunnel motor off. All flow incident on the tail surfaces is due to the propeller wash. The propeller was driven at 3000, 4000, 5000, and $6000 \mathrm{rpm}$ based on measurements taken by an onboard commutation sensor connected to the motor. The revolutions per minute were held constant within $0.5 \%$ error for each test point. The tunnel test-section access door was left open to minimize test section flow driven by the propeller. This allows the pressure and FT instrumentation to be compared in an approximately steady hover condition. Pressure measurements from the distributed instrumentation suite indicate that the prop-wash covers the entire tail section during these tests.

The flow direction measurements taken by the prop-wash probe during the hover tests are summarized in Table 2 . The flow angles appear to diminish with increasing revolutions per minute and axial velocity. The proposed reformulation of the steady-flight equations does not consider the swirl imparted by the propeller, and so these measurements are not used in the presented analysis.

\section{Hover Pitch Moment}

Pitch control in a hover is achieved through the use of the conventional elevators operating in the high-speed propeller wash. A series of elevator sweeps were conducted at the four test revolutions per minute. The conditions encountered for these tests are summarized in Table 3 . Note that the wind tunnel is not climatecontrolled; thus, air temperature is influenced by ambient temperature in Michigan at the time of testing.

The air density is calculated from temperature and barometric pressure measurements assuming dry air. Following the axis convention, positive elevator deflections and pitching moments correspond to a nose-up moment. Both sets of instrumentation show general agreement. As expected, pitch moments vary with increasing propeller revolutions per minute. With increasing propeller revolutions per minute and unsteady but periodically varying (quasisteady) flow, the standard deviations for both sets of measurements are larger. Pressure-based estimates are noted to be more susceptible to noise. The pitch moment data are summarized in Fig. $\underline{6}$.

Both sets of instrumentation show that control moments are linear between a -25 and 25 deg elevator deflection range with a slight decrease in slope at larger deflections angles. The pressurebased instrumentation consistently underestimates the measurements reported by the FT sensor, showing what appears to be a shallower slope. It is hypothesized that this is due to a consistent underestimation of the aerodynamic forces by the discretization scheme because no pressure ports are located near the leading edge of the control surfaces where local pressure peaks are anticipated. This is explored next.

\section{Yaw Moment Comparisons}

As with the elevator, the rudder is used to command yaw moments when in a vertical hover. A series of yaw-only sweeps at the same propeller revolutions per minute were carried out under conditions summarized in Table 4 .

As with the pitch moments and elevator deflections, convention is followed where positive rudder deflections result in positive yaw moments that point the nose of the aircraft to starboard. Both sets of instrumentation show general agreement. As expected, yaw moments scale with increasing propeller revolutions per minute.

Table 2 Hover test prop-wash probe mesaurements for elevator sweeps

\begin{tabular}{ccccc}
\hline \hline Prop revolutions per minute & $\alpha_{\mathrm{pw}}, \operatorname{deg}$ & $\sigma$ & $\beta_{\mathrm{pw}}, \mathrm{deg}$ & $\sigma$ \\
\hline 3000 & -4.17 & 4.3 & -11.93 & 1.9 \\
4000 & -2.03 & 4.5 & -11.46 & 2.1 \\
5000 & -1.74 & 4.8 & -10.65 & 2.3 \\
6000 & -1.27 & 4.7 & -9.61 & 2.4 \\
\hline \hline
\end{tabular}


Table 3 Hover test conditions for elevator sweeps

\begin{tabular}{|c|c|c|c|c|}
\hline $\begin{array}{c}\text { Elevator test } \\
\text { case, rpm }\end{array}$ & $\begin{array}{c}\text { Average } \\
V_{\mathrm{pw}}, \mathrm{m} / \mathrm{s} \\
\end{array}$ & $\begin{array}{c}\text { Air } \\
\text { temperature, } \\
{ }^{\circ} \mathrm{C} \\
\end{array}$ & $\begin{array}{c}\text { Barometric } \\
\text { pressure, inHg }\end{array}$ & $\begin{array}{c}\text { Computed air } \\
\text { density, } \mathrm{kg} / \mathrm{m}^{3}\end{array}$ \\
\hline 3000 & 9.1 & 3 & 29.5 & 1.26 \\
\hline 4000 & 11.7 & 8 & 29.4 & 1.23 \\
\hline 5000 & 14.71 & 8 & 29.4 & 1.23 \\
\hline 6000 & 17.81 & 3 & 29.5 & 1.26 \\
\hline
\end{tabular}

With increasing propeller revolutions per minute, the standard deviations for both sets of measurements are larger for yaw than for pitch. Pressure-based estimates are noted to be more susceptible to noise than FT data as with the pitch moment results but with fewer sensors and less cumulative measurement error; the standard deviation intervals of the aerodynamic yaw moment measurements were also smaller than for the pitch measurements. The yaw results are summarized in Fig. 7.

Both sets of instrumentation show that yaw moments are approximately linear in control surface deflection over a -25 and 25 deg range with a slight decrease in slope at larger deflections angles. As with the pitch moments, the pressure-based instrumentation consistently underestimated the yaw moments measurements reported by the FT sensor. The results show a larger discrepancy than that encountered with the pitch data, which may be due to the yaw moment contributions from the uninstrumented vertical sides of the fuselage.

\section{B. Combined Freestream and Prop-Wash Cases}

Hover is a special case where the prop-wash is directed over the tail surfaces and is aligned with the fuselage. The flow conditions encountered during flight operations at high angles of attack are often
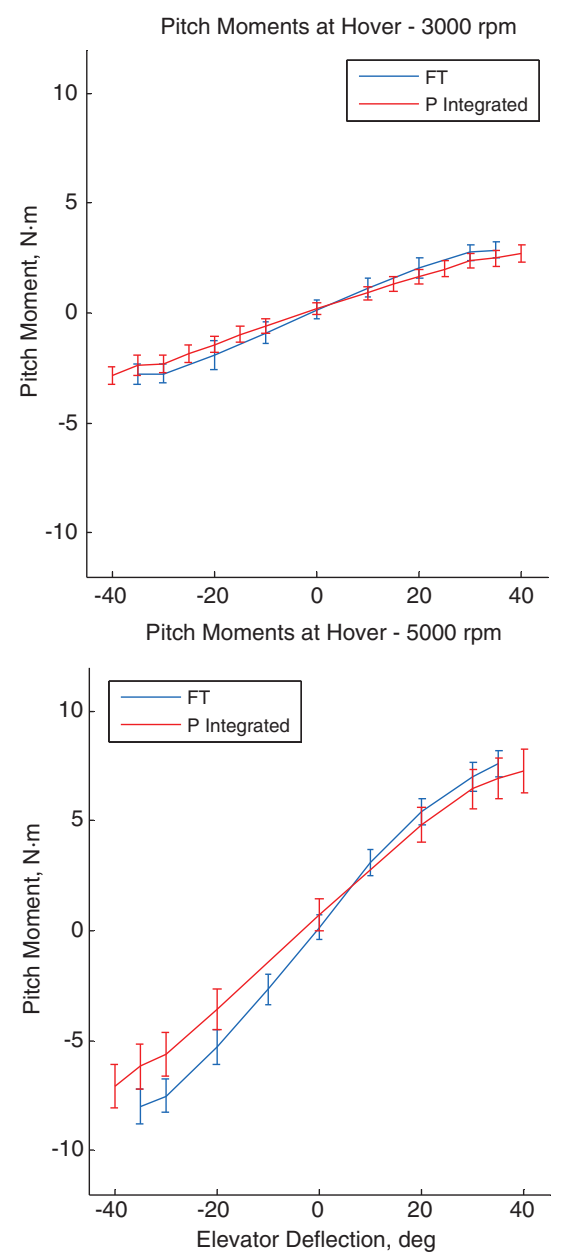

Fig. 6 Pitch moment measurements at hover. more complex with an incident freestream that can interact with the prop-wash. Two test cases were run to evaluate the instrumentation package beyond hover. The first is a simulated level cruise condition where the freestream velocity vector is aligned with the thrust line. The second case represents a low-airspeed, postwing stall condition near hover. These two exploratory test cases were chosen to work within the constraints of the wind-tunnel test section. The main goal is providing data to evaluate the aerodynamic sensing scheme under mixed flow conditions.

\section{Test Case 5kCruise: Forward-Flight Results}

A $5000 \mathrm{rpm}$ cruise flight test case approximates a flight condition where the incident freestream is substantial but still slower than the prop-wash probe airspeed accounting for flow conditions aft of the propeller. This test provides a mixed flow environment across the tail surfaces where there is a significant degree of uniform flow over the airframe in addition to the incident propeller wash. The propeller is set to $5000 \mathrm{rpm}$ with the aircraft mounted level in a freestream of $12 \mathrm{~m} / \mathrm{s}$. Conditions encountered during the $5 \mathrm{kCruise}$ test case are summarized in Table $\underline{5}$.

With the addition of a low-turbulence freestream, both FT and pressure measurements show smaller standard deviation intervals. Note also that the control moments are significantly larger for the same surface deflections when at hover. This is due to the faster and more uniform velocity over the entire tail section driven by the freestream. The plots in Fig. $\underline{8}$ summarize these results.

Although the velocities reported by the prop-wash probe are only slightly larger than those encountered at the $6000 \mathrm{rpm}$ hover case, it is clear that the resulting moments are significantly larger. This indicates that prop-wash probe measurements alone are not sufficient in describing the flow conditions over the tail under all flight conditions. During flight operations that cross different flight
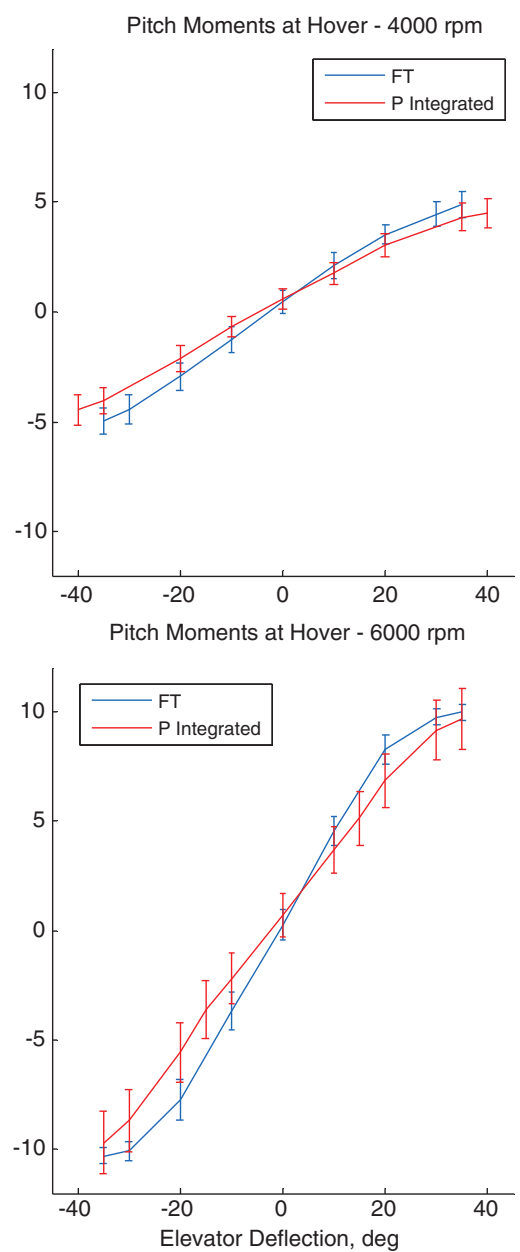
Table 4 Hover test conditions for rudder sweeps

\begin{tabular}{ccccc}
\hline \hline & \multicolumn{4}{c}{ Air } \\
$\begin{array}{c}\text { Rudder test } \\
\text { case, rpm }\end{array}$ & $\begin{array}{c}\text { Average } \\
V_{\mathrm{pw}}, \mathrm{m} / \mathrm{s}\end{array}$ & $\begin{array}{c}{ }^{\circ} \mathrm{C} \\
\text { temperature, }\end{array}$ & $\begin{array}{c}\text { Barometric } \\
\text { pressure, inHg }\end{array}$ & $\begin{array}{c}\text { Computed air } \\
\text { density, } \mathrm{kg} / \mathrm{m}^{3}\end{array}$ \\
\hline 3000 & 9.4 & 1 & 29.5 & 1.27 \\
4000 & 11.93 & 11 & 29.4 & 1.22 \\
5000 & 14.61 & 11 & 29.4 & 1.22 \\
6000 & 17.81 & 1 & 29.5 & 1.27 \\
\hline \hline
\end{tabular}

regimes, the information from the prop-wash probe will still need to be augmented by conventional aircraft wind vector measurements, or else model coefficients will need to be scheduled by freestream velocity conditions rather than strictly using prop-wash information. Under these larger flow velocities, the distributed pressure sensing scheme still provides moment estimates that agree with those measured by the FT sensor. The moments remain linear with control surface defection, and the pressure-based slope is again seen to be shallower than the slope given by the FT data.

\section{Test Case Alpha25: Low-Speed Nonzero-Angle-of-Attack Case}

In the cruise case, the incident freestream is aligned with the propeller wash, resulting in relatively simple interaction. During operations at low freestream airspeed, large angles of attack up to 50 deg have been encountered that can dramatically change the local flow conditions across the tail surfaces. The conditions in this test case aim to represent some of these challenges, with the $25 \mathrm{deg}$ angle of attack selected based on wind-tunnel test section size constraints. For this test set, the aircraft is mounted at a $25 \mathrm{deg}$ angle with respect
Table 5 Test conditions for the $5 \mathrm{kCruise}$ case

\begin{tabular}{|c|c|c|c|c|c|}
\hline $\begin{array}{l}5 k \text { Cruise } \\
\text { test case }\end{array}$ & $\begin{array}{l}\text { Average } \\
V_{\mathrm{ac}}, \mathrm{m} / \mathrm{s}\end{array}$ & $\begin{array}{c}\text { Average } \\
V_{\mathrm{pw}}, \\
\mathrm{m} / \mathrm{s}\end{array}$ & $\begin{array}{c}\text { Air } \\
\text { temperature, } \\
{ }^{\circ} \mathrm{C} \\
\end{array}$ & $\begin{array}{c}\text { Barometric } \\
\text { pressure, } \\
\text { in } \mathrm{Hg}\end{array}$ & $\begin{array}{c}\text { Computed } \\
\text { air density, } \\
\mathrm{kg} / \mathrm{m}^{3}\end{array}$ \\
\hline Pitch & 12.31 & 18.24 & 3 & 29.1 & 1.24 \\
\hline Yaw & 12.35 & 19.32 & 3 & 29.1 & 1.24 \\
\hline
\end{tabular}

to the test section, and the door is shut. The propeller is driven at $5000 \mathrm{rpm}$, resulting in a small but measurable freestream through the test section. As an added complication, the tail surfaces are placed near the floor of the test section and are subject to wall interactions. The test conditions are presented in Table $\underline{6}$.

With the propeller driven at $5000 \mathrm{rpm}$, the measured prop-wash velocities are comparable to those encountered in the previous test case with a large freestream. This is likely due to the significant test section blockage caused by the model when mounted at $25 \mathrm{deg}$, producing a localized region of accelerated flow near the model. This does not affect the comparison because both sets of instrumentation are subject to the same flow conditions, making for a valid comparison. The results of the Alpha25 test case are plotted in Fig. 9.

The effect of wall interaction can be more clearly observed in both the yaw and pitch moment data. At rudder deflections between -40 and $-15 \mathrm{deg}$, a change in slope is observed for negative rudder deflections. This directionality is likely due to the clockwise swirl caused by the propeller interacting with the test section floor, thus affecting the flow angle of attack on the rudder. As with all the previous cases, the aerodynamic instrumentation is seen to consistently underestimate the resulting moments.
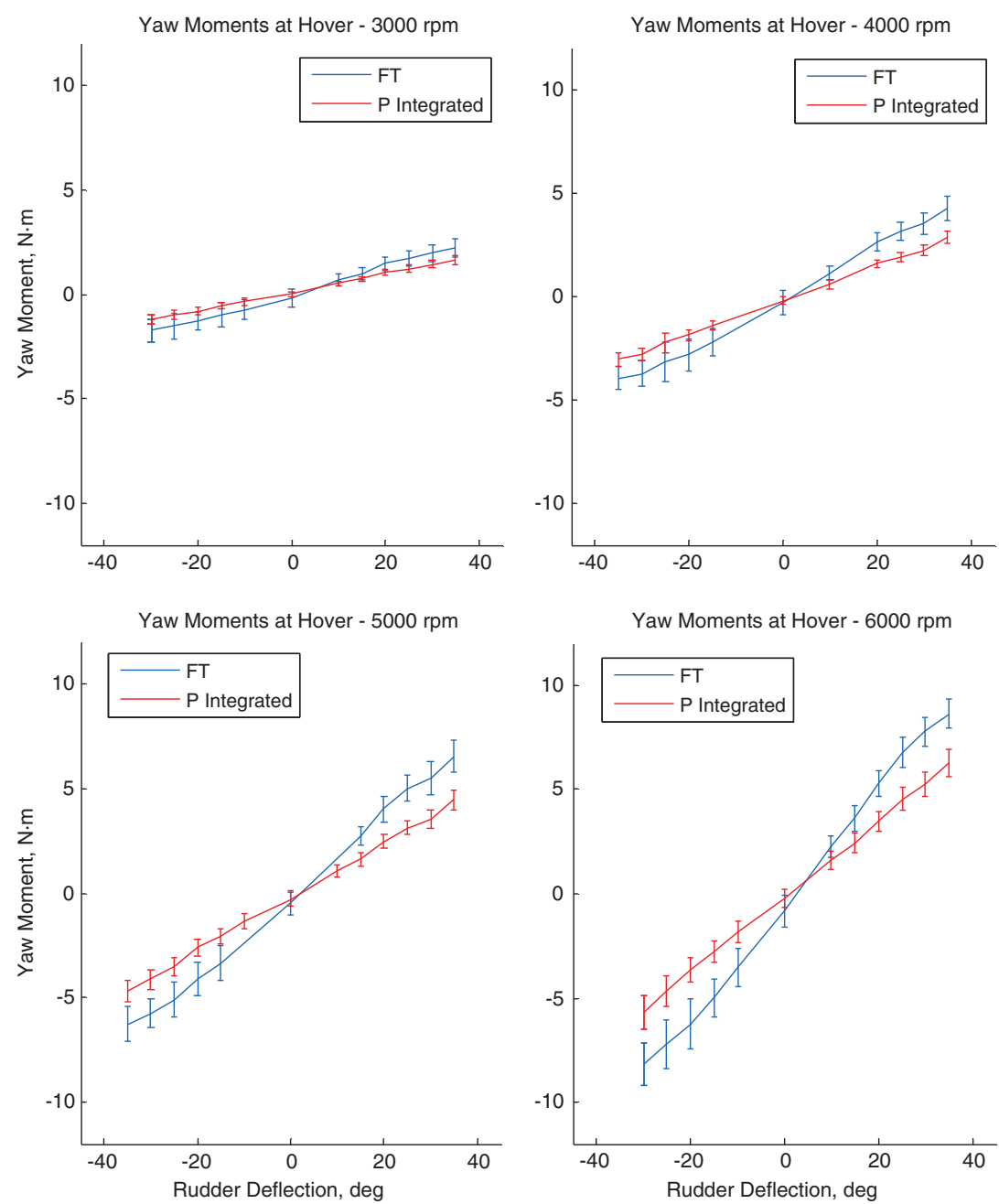

Fig. 7 Yaw moment measurements at hover. 

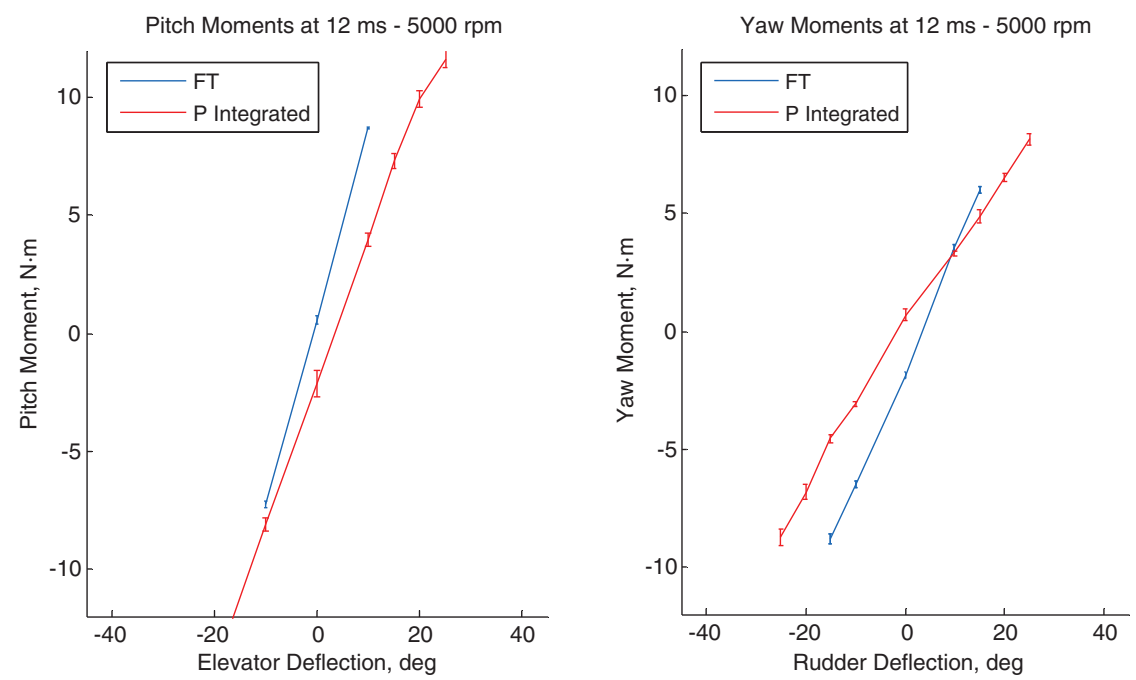

Fig. 8 Pitch and yaw moment measurements at $12 \mathrm{~m} / \mathrm{s}$ and $5000 \mathrm{rpm}$.

\section{Independence of Pitch and Yaw Control Surfaces}

In the steady-flight equations, it is assumed that elevator and rudder commands are independent of each other such that elevator deflections do not affect yaw moments, and rudder deflections do not impact pitch moments. A series of tests were run to estimate the effect of elevator commands on the yaw moments when in a hover condition. This would determine if the reformulated flight equations for steady hover and high-angle-of-attack flight will require additional terms that account for this effect.

A rudder sweep was performed at a number of elevator deflections angles for each of the thrust settings, and the yaw moments measured by the FT sensor are compared. Sample results at 5000 and $6000 \mathrm{rpm}$ are plotted in Fig. 10.

Throughout the linear range of elevator deflection between -30 and $30 \mathrm{deg}$, the yaw moments resulting from rudder commands do not vary appreciably. This shows that the elevator does not affect the yaw moments across the test range. The data from these tests allow a limited characterization of the reverse relationship; two sample plots at 3000 and $6000 \mathrm{rpm}$ are shown in Fig. 11, depicting elevator sweeps with changing rudder. It can be observed that, throughout the range of 30 and 30 deg of rudder deflection, the relationship between elevator commands and pitch moments remains the same. This validates the assumption of decoupled pitch and yaw moment control in a hover.

\section{Experimental Coefficient Determination}

Pitch and yaw measurements from a series of wind-tunnel-based tests have been presented. Measurements from the aerodynamic sensing system were filtered using a simple moving average filter with a period equal to two propeller revolutions. This is representative of the quality of data available in flight. The FT measurements were time averaged. Data from all the test cases indicate largely linear relationships between control surface deflections and the aerodynamic moments associated with them. A baseline comparison between independent FT and pressure-based measurements indicate that the aerodynamic data system slightly underestimates the moments measured by the FT sensor. This discrepancy is discussed further next. Airspeeds measured by the prop-wash probe increase with increasing propeller revolutions per

Table 6 Test conditions for the Alpha25 case

\begin{tabular}{lccccc}
\hline \hline Alpha25 & $\begin{array}{c}\text { Average } \\
V_{\text {ac }}, \mathrm{m} / \mathrm{s}\end{array}$ & $\begin{array}{c}\text { Average } \\
V_{\mathrm{pw}}, \\
\mathrm{m} / \mathrm{s}\end{array}$ & $\begin{array}{c}\text { Air } \\
\text { temperature, } \\
{ }^{\circ} \mathrm{C}\end{array}$ & $\begin{array}{c}\text { Barometric } \\
\text { pressure, } \\
\text { inHg }\end{array}$ & $\begin{array}{c}\text { Air } \\
\text { density, } \\
\mathrm{kg} / \mathrm{m}^{3}\end{array}$ \\
\hline Pitch & 2.05 & 17.02 & 5 & 29.2 & 1.24 \\
Yaw & 2.01 & 16.9 & 1 & 29.5 & 1.27 \\
\hline \hline
\end{tabular}

minute as expected, providing data that can be used for real-time flow characterization over the tail, potentially in conjunction with freestream flow measurements from the traditional wing-mounted airdata probe, given mixed flow conditions.

\section{A. Linear Moment Model Development for Hover}

Although the poststall flight regime is nonlinear with respect to lift and drag, it can be observed from the data that the pitch and yaw moment response to changing elevator and rudder deflections are largely linear when in a hover. This allows a linear moment model to be established based on experimental results and additional wind vector measurements (from the prop-wash probe) even at zero vehicle airspeed. This section presents pitch and yaw moment coefficients extracted from Funtana hover test data as normalized by measured propeller-wash velocity.

The resulting model is compared against test data, and the limitations of the linear approximation are discussed, also motivating the additional use of distributed pressure sensing. An empirical, quadratic relationship between measured velocity and propeller revolutions per minute for the range of test conditions is presented in Fig. 12, which allows a simple revolutions-per-minute-based correlation to be performed for the tested propeller if desired.

\section{Moment Coefficients for Use with $V_{\mathrm{pw}}$ in Hover}

With the additional prop-wash airspeed measurement and the moment measurements from the FT sensor, the new pitch and yaw coefficients for the reformulated steady-flight equations from Eqs. (13) and (16) can be determined. When in a hover, the freestream airspeed $V_{\text {ac }}$ will approach 0 . The pitch and yaw equations then become

$$
\begin{gathered}
M_{h \text { over }}=\frac{1}{2} \rho V_{\mathrm{pw}}^{2} S_{h \text { tail }} l_{\text {tail }} C_{M_{h \text { tail }}} \\
N_{h \text { over }}=\frac{1}{2} \rho V_{\mathrm{pw}}^{2} S_{v \text { tail }} l_{\text {tail }} C_{N_{v \text { tail }}}
\end{gathered}
$$

Because basic air data systems are only capable of measuring $V_{\mathrm{ac}}$, this formulation represents the ability for feedback that is not available without additional instrumentation developed in this work. Through estimates of dynamic pressure $\frac{1}{2} \rho V_{\mathrm{pw}}^{2}$ over the tail surfaces, the additional prop-wash probe measurement $V_{\mathrm{pw}}$ allows determination of the moments in hover. In a fixed flight condition such as hover, the incident flow angle due to the propeller wash is approximately constant. This allows both terms to be treated as a single coefficient in hover, simplifying Eqs. (15) and (18) to 

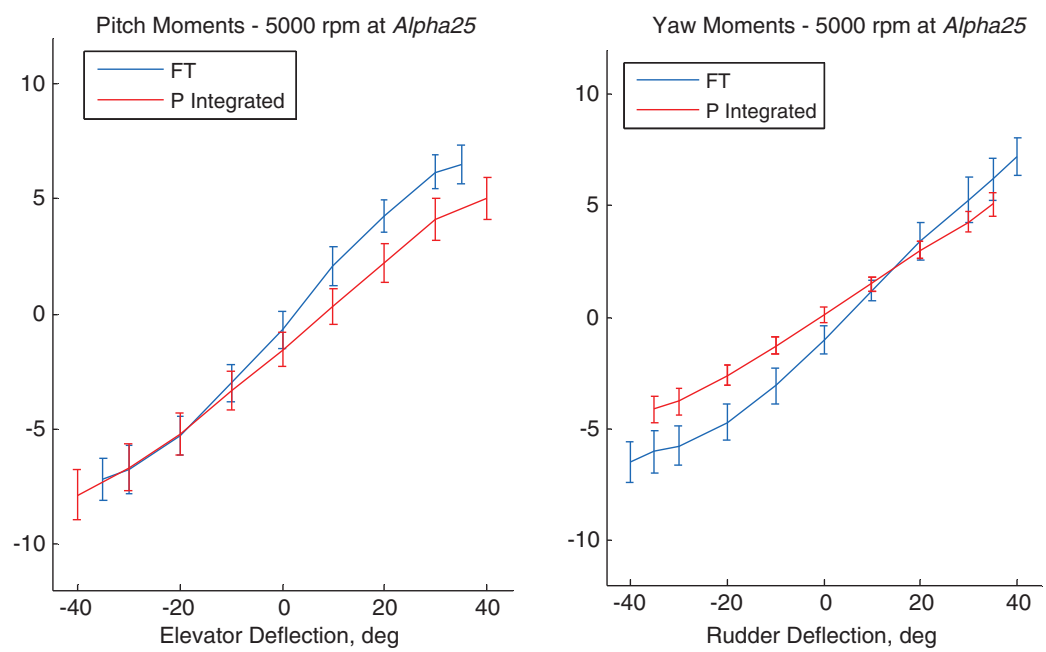

Fig. 9 Pitch and yaw moments from the Alpha25 test case.
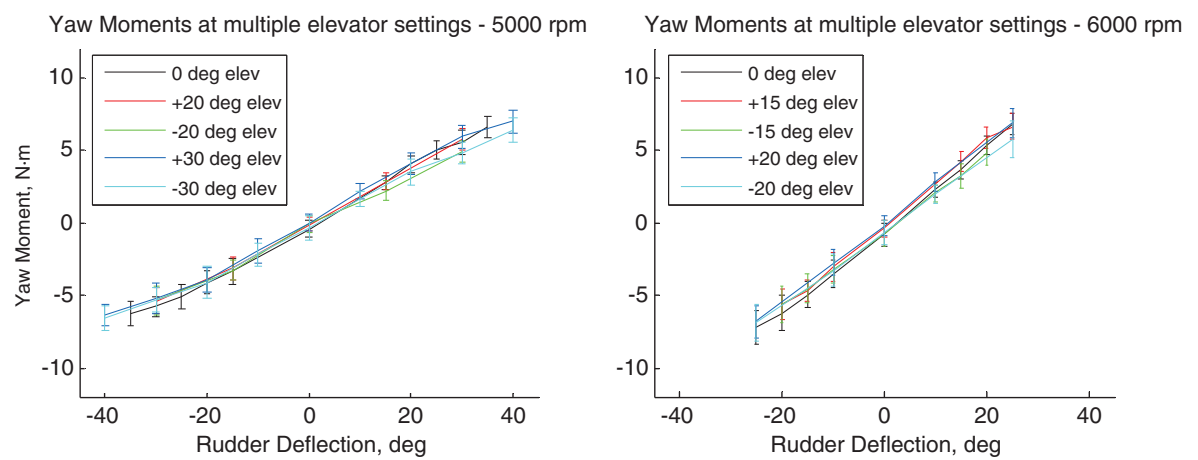

Fig. 10 Combined elevator and rudder deflection test cases at 5000 and $6000 \mathrm{rpm}$ showing negligible cross coupling between elevator and yaw moments.

$$
\begin{gathered}
C_{M_{h t \text { tail }}}=C_{M_{h \text { tail }_{-} h \text { over }}}+C_{M_{\text {htail }_{\delta_{e}}}} \delta_{e} \\
C_{N_{\text {vtail }}}=C_{N_{\text {vtail }_{0} \text { hover }}}+C_{N_{\text {vtail }_{\delta_{r}}}} \delta_{r}
\end{gathered}
$$

where the coefficients are determined from the hover test series by normalizing the pitch or yaw moment data by tail surface area and by measured local dynamic pressure. Once the data have been normalized, a linear fit across the linear range of the data for the hover coefficients is obtained. The plots in Fig. 13 show normalized pitch and yaw moment data and the resulting linear fit for each axis. The trend lines for the cases collapse when normalized and show strong linear trends. The resulting coefficients and $R^{2}$ correlation values are summarized in Table 7.

Through the described methodology, the coefficients in Eqs. (28) and (29) can be determined. These represent a linear aerodynamic model similar to the basic steady-flight equations that is made possible through an additional velocity measurement. Note that these coefficients are valid only for a range of operating conditions where
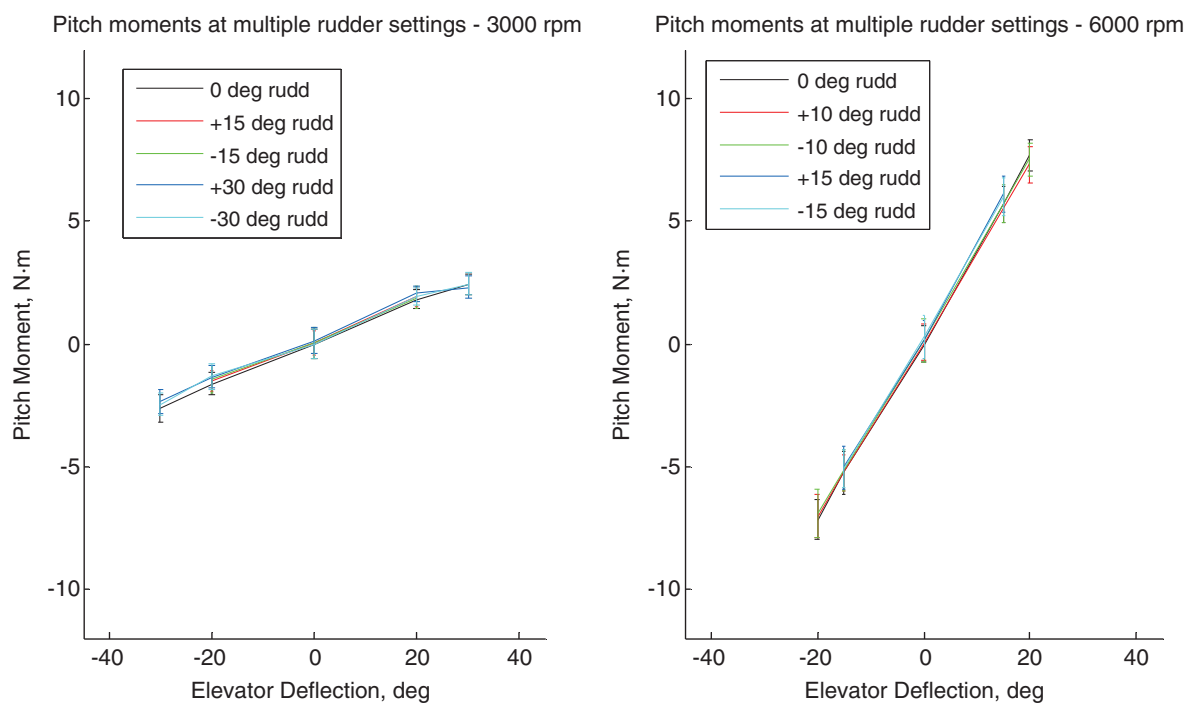

Fig. 11 Combined rudder and elevator deflection test cases showing negligible cross coupling between rudder and pitch moments. 
aircraft airspeed is negligible and the prop-wash is approximately aligned with the fuselage.

\section{Further Investigation of the Linear Aerodynamics Model}

As with the original steady-flight equations, the model described previously is only valid in conditions for which a linear model is sufficient. Key coefficients are determined by only considering data within the ranges of control surface deflections for which linear curve fits adequately approximate resulting moments. This section compares the output of the linear model against actual test data and discusses its performance under the different test conditions.

a. Validation of the Linear Model for Hover.-Estimates of the linear model are compared against the normalized pitch and yaw FT data encountered in hover across all test conditions. Results are shown in Fig. 14. Based on this comparison, the pitch and yaw moment linear models are valid throughout a large range of the hover data. The linear models tend to overestimate moments due to deflections larger than $20 \mathrm{deg}$. All predictions are accurate for 4000, 5000, and $6000 \mathrm{rpm}$ cases so long as the control surface deflection is 20 deg or less. Pitch estimates are also consistently accurate for the $3000 \mathrm{rpm}$ case.

Note that, in a $3000 \mathrm{rpm}$ hover case, the test data show a noticeable asymmetry in control authority for left-rudder commands. This is likely due to the relatively more pronounced clockwise flow structure at prop-wash velocities. Because the linear model is a compilation of all hover data, this asymmetry is not reflected in its output. To summarize, when in a hover, the coefficient-based formulation of the reformulated steady-flight equations generally work well in reproducing the pitch and yaw relationships that were used to determine its coefficients when an additional prop-wash velocity measurement is available.

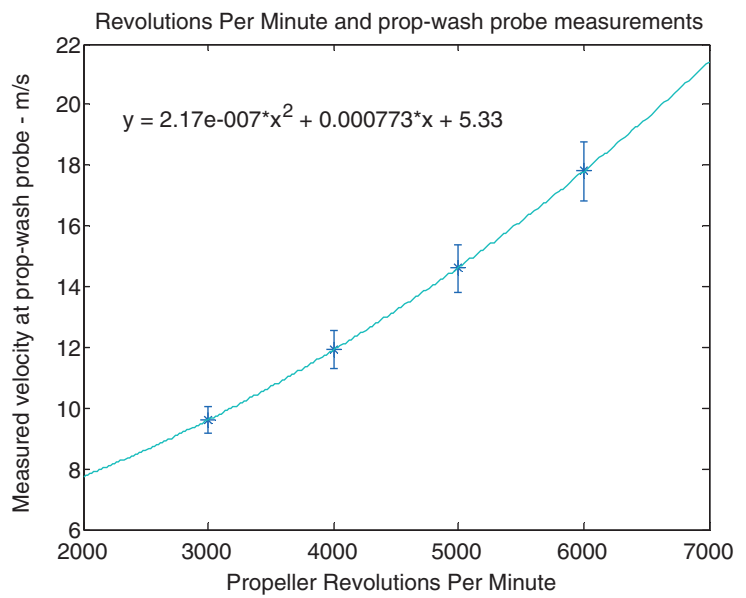

Fig. 12 Empirical relationship between measured revolutions per minute and measured prop-wash velocity.

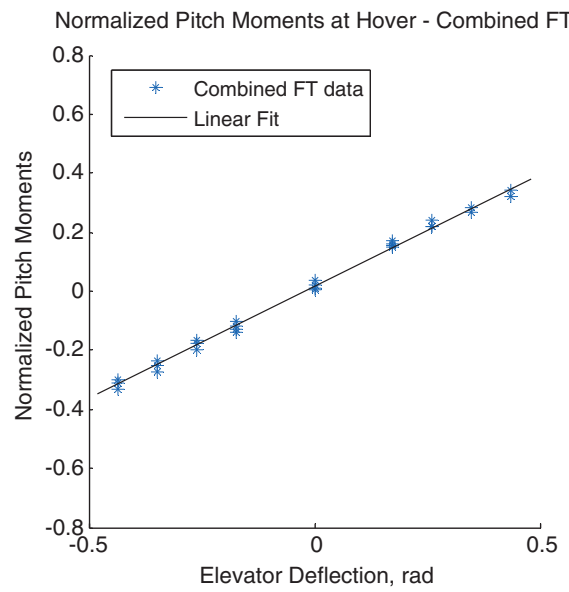

Table 7 Linear aerodynamic coefficients computed from hover data

\begin{tabular}{lccc}
\hline \hline Axis & $C_{\text {moment }_{0} \text { hover }}$ & $C_{\text {moment }_{\delta}}$ & $R^{2}$ \\
\hline Pitch $M$ & 0.01716 & 0.7611 & 0.996 \\
Yaw $N$ & -0.01667 & 0.9978 & 0.983 \\
\hline \hline
\end{tabular}

b. Linear Model Applicability Near Hover.-The linearized moment model developed at hover is further investigated at the slower freestream conditions $(2 \mathrm{~m} / \mathrm{s})$ established for the high-alpha windtunnel test series discussed previously. By comparing the output of the linear model to the Alpha25 case, the linear model can be assessed outside of the data that were used in its formulation, representing a "high-angle, slow flight" condition that might be quite useful for small UAS flight operations. In Alpha25 configuration, the testsection flow that is incident on the aircraft at a nonzero angle introduces a degree of freestream interaction with the prop-wash. Although the mounting angle of the aircraft is large, the incident freestream is small, which thus simulates an aircraft that is maneuvering near hover. When in this flight condition, the contribution of the pitch and yaw moments from the fuselage and wings will be nonzero. Because the coefficients $C_{M_{\mathrm{ac}}}$ and $C_{N_{\mathrm{ac}}}$ from Eqs. (13) and (16) are still being determined as part of ongoing work, this comparison will focus only on the slope predicted by the linear model and propwash probe velocity $V_{\mathrm{pw}}$. The comparison between acquired FT data for Alpha25 and estimates from the models linearized from hover are shown in Fig. 15 for both pitch and yaw. In these plots, the data are compared in natural moment units because a suitable velocity scale has not been established for the Alpha25 test case.

The linear model is capable of predicting the general trends but does not precisely align, particularly in pitch. The difference at small elevator deflections, on the order of $1.5 \mathrm{~N} \cdot \mathrm{m}$, is likely still adequate for use in a feedback control model. The linear model predicts yaw moments adequately until nonlinear effects are encountered at large rudder deflections. However, the slope of the pitch data is noticeably different from the slope encountered in hover, suggesting that a new set of coefficients will be required even for small freestream flow velocities.

\section{B. Embedded Pressure Sensing Scheme}

An embedded pressure sensing scheme to directly measure aerodynamic lift on the tail surfaces offers potential improvement over the linear modeling approach presented previously. Distributed sensing across the tail section allows pressures to be measured across the stabilizer and control surfaces. This allows the actual aerodynamic moments induced by the tail to be measured in flight. By taking measurements instead of relying on models, information about complex flow conditions such as those due to fuselage effects and the propeller wash being redirected away from the tail section can be more accurately gathered for the future purpose of flight control.

Fig. 13 Compiled FT data from hover cases and resulting linear fit.

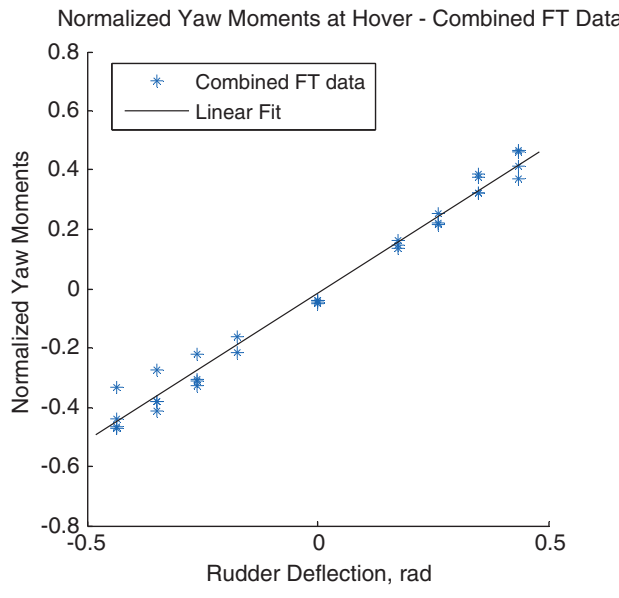



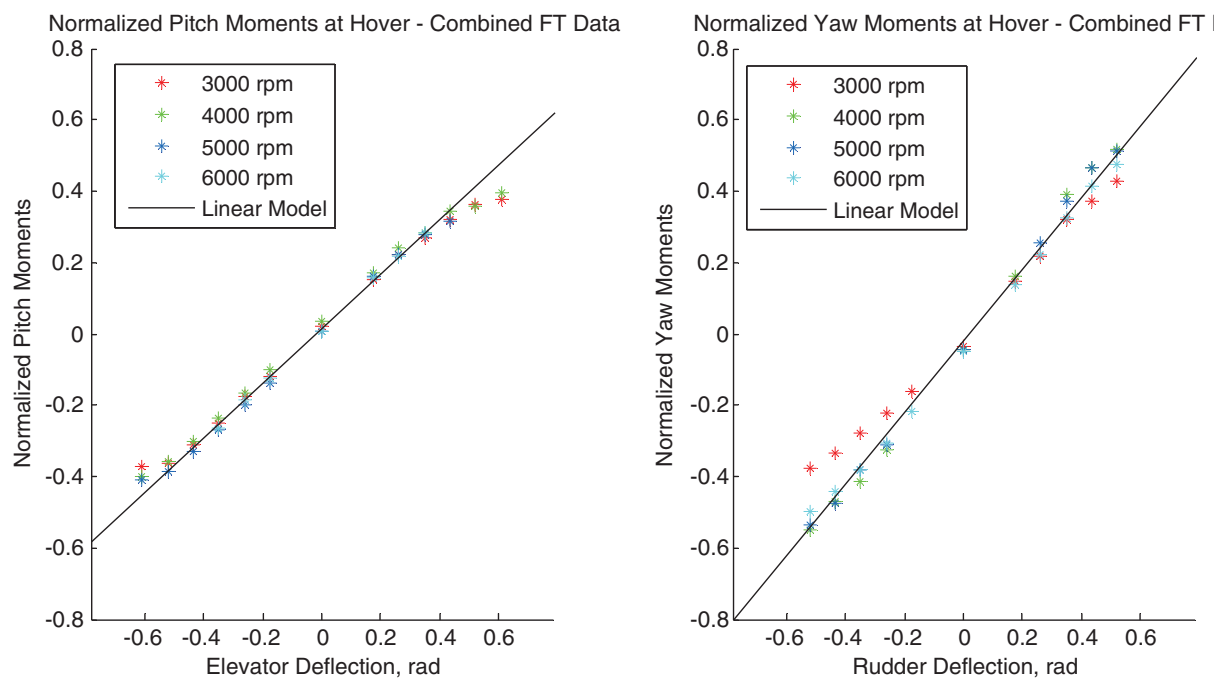

Fig. 14 Comparison between linear model and compiled hover data.
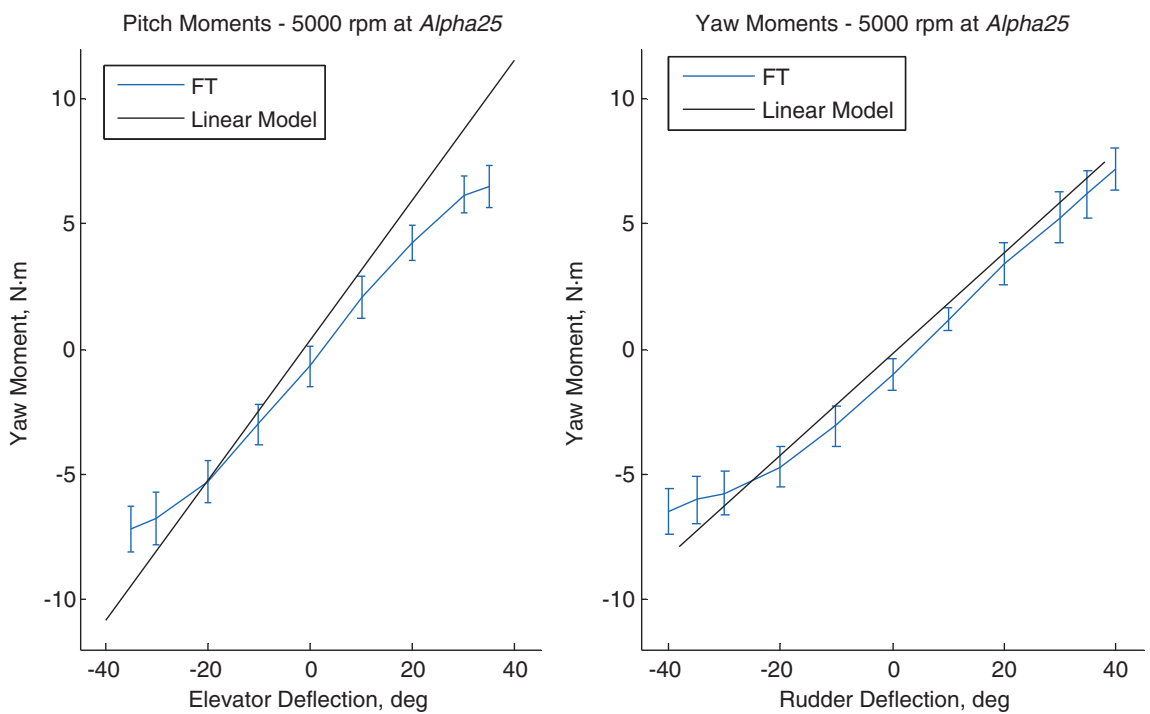

Fig. 15 Linear model compared with Alpha25 test case results.

Differential pressure $P_{\text {diff- } i}$ is measured across the horizontal tail, elevators, vertical tail, and rudder at $n$ locations. Each location $i$ corresponds to a panel of area $S_{h \text { tail } i}$ that is $l_{i}$ from the center of gravity and has a deflection angle of $\theta_{i}$. This deflection angle depends on the panel orientation relative to the surface reference angle in body-fixed axes and is approximately zero for panels on the fixed stabilizers and equal to the control surface deflection angle for panels on the rudder and elevator. As previously described in Eqs. (23) and (24), the terms relating to the control surfaces can be rewritten using terms determined through the onboard instrumentation.

This represents the ability to "close the loop" with regard to the aerodynamics in complex flow conditions in a manner not possible with traditional air data systems. Initial comparisons have shown that the raw data show general agreement, but additional calibration is required. The following subsections propose a calibration and correction methodology to enable the embedded pressure measurements to be used to better characterize the moments. The calibration process is first described, and the performance of the corrected aerodynamic sensing system is then evaluated.

\section{Calibration of Pressure Data Integration Using a Low-Turbulence Reference Case}

A low turbulence test case can provide a uniform flowfield without the predominant swirl and unsteady effects of the propeller wash.
A reference test case was run by using the freestream generated by the wind tunnel with the propeller fixed (zero thrust). The chosen airspeed of $12 \mathrm{~m} / \mathrm{s}$ (measured as an actual wind-tunnel test speed of $12.3 \mathrm{~m} / \mathrm{s}$ ) represents a low-speed cruise condition for the flight vehicle. The applicable equations are

$$
\begin{gathered}
M=\frac{1}{2} \rho V_{\mathrm{ac}}^{2} S b C_{M_{\mathrm{ac}}}+A_{M} \sum_{i=1}^{\mathrm{n}_{h \text { tail }}} \cos \theta_{i} \cdot P_{\text {diff }-i} \cdot S_{h \text { tail } i} \cdot l_{i} \\
N=\frac{1}{2} \rho V_{\mathrm{ac}}^{2} S c C_{N_{\mathrm{ac}}}+A_{N} \sum_{i=1}^{n_{\text {vtail }}} \cos \theta_{i} \cdot P_{\text {diff }-i} \cdot S_{\text {vtail } i} \cdot l_{i}
\end{gathered}
$$

where the coefficients $A_{M}$ and $A_{N}$ are sought to improve pressurebased estimation of pitch and yaw moments. Basic rudder and elevator sweeps are completed, and measurements from both sets of instrumentation are compared under low-turbulence flow conditions. No additional filtering is performed for the pressure measurements because the propeller is not rotating. These results are shown in Fig. 16; note that the FT data for pitching moment are truncated once the sensor saturates between 15 and 20 deg elevator deflection. The comparison for the low-turbulence test case shows the same trends as in the other conditions, indicating that observed discrepancies are due to issues in calibration rather than unsteady flow generated by the 
Pitch Moments - 12 ms freestream only

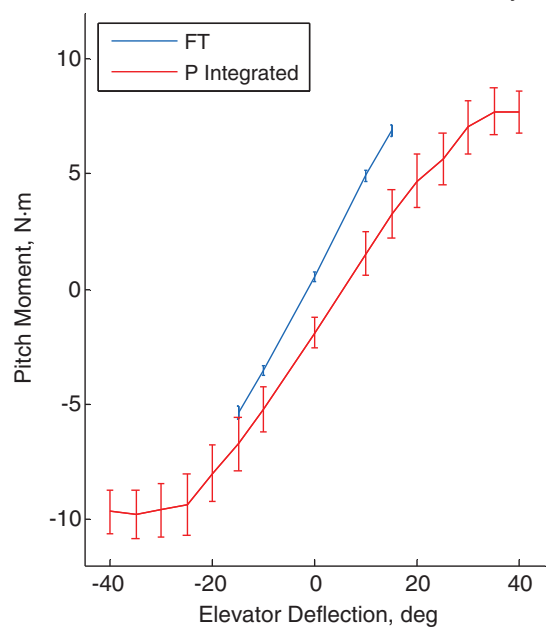

Yaw moments 13 ms freestream only

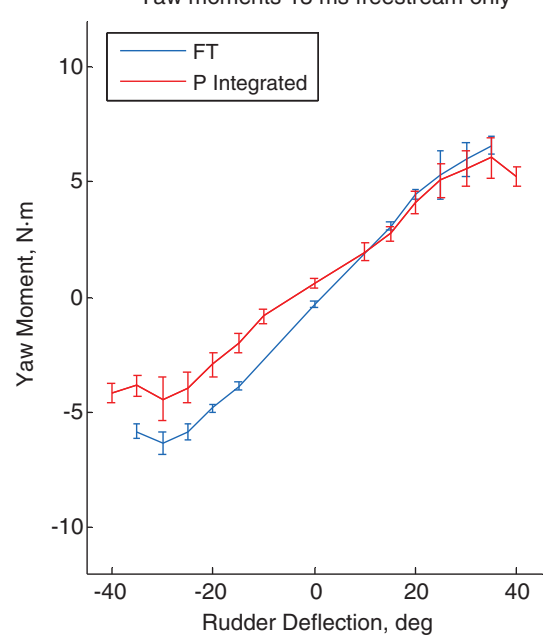

Fig. 16 Low-turbulence freestream training data set.

propeller. In these plots, the data are presented in natural units because the results are used to calibrate the tail instrumentation. The pressure instrumentation gives moment measurements that follow the same trend but are smaller than those measured by the FT sensor. An offset with the control surfaces centered can also be observed. This is most likely due to the moments contributed by the aircraft fuselage and wing and described by coefficients $C_{M_{\mathrm{ac}}}$ and $C_{N_{\mathrm{ac}}}$ in the reformulated equations. Further testing to quantify these coefficients to form a complete aerodynamic model is described as future work.

Using these results as a training case, the coefficients $A_{M}$ and $A_{N}$ are computed by comparing the linear slopes of the pressureintegrated and FT sensor measurements. The linear slopes for each axis is computed by first choosing two control surface deflection angles $\theta_{1}$ and $\theta_{2}$ on either side of a neutral setting that correspond to moments that are within the linear range of the data. Each $\theta_{i}$ will have two corresponding moment measurements from the aerodynamic sensing system and the FT sensor, denoted as $\mathrm{Mom}_{\mathrm{Aero}, i}$ and $\mathrm{Mom}_{\mathrm{FT}, i}$, respectively. The computation is given in Eqs. (34-36):

$$
\begin{gathered}
\text { Slope }_{\mathrm{Aero} M, N}=\frac{\operatorname{Mom}_{\mathrm{Aero}, 2_{M, N}}-\operatorname{Mom}_{\mathrm{Aero}, 1_{M, N}}}{\theta_{2_{M, N}}-\theta_{1_{M, N}}} \\
\text { Slope }_{\mathrm{FT}_{M, N}}=\frac{\operatorname{Mom}_{\mathrm{FT}, 2_{M, N}}-\operatorname{Mom}_{\mathrm{FT}, 1_{M, N}}}{\theta_{2_{M, N}}-\theta_{1_{M, N}}} \\
A_{M, N}=\frac{\text { Slope }_{\mathrm{FT} M, N}}{\text { Slope }_{\text {Aero } M, N}}
\end{gathered}
$$

Estimates of the linear slopes and the coefficients for pitch and yaw are summarized in Table $\underline{8}$.

\section{Calibration Applied to Hover Data}

The calibration terms calculated using the equations and parameters from the previous was applied to the propeller-driven test cases. As shown in Figs. 17 and $\underline{18}$, the updated pressure-based moment measurements show good agreement with the FT measurements with overlapping standard deviation intervals at all

Table 8 Estimated linear slopes from low-turbulence training case

\begin{tabular}{lccc}
\hline \hline Axis & Slope $_{\text {Aero } M . N}$ & Slope $_{\mathrm{FTM}, N}$ & $A_{M, N}$ \\
\hline Pitch $M$ & 19.589 & 24.549 & 1.253 \\
Yaw $N$ & 9.183 & 13.459 & 1.466 \\
\hline \hline
\end{tabular}

data points. Corrected pitch moments at hover are plotted in Fig. 17, followed by corrected yaw moments at hover in Fig. 18. In these plots, the results are presented in natural moment to reflect their applicability in flight conditions not suited to a conventional coefficient-based approach. Based on these results, it can be concluded that the calibration factors computed in the lowturbulence test case applies to steady hover flight conditions. In contrast to the linear coefficients computed previously, the distributed sensor scheme measurements corrected by the instrumentation calibration are also applicable throughout significantly different flight regimes.

The asymmetry in yaw coefficient that was noted in the FT measurements at $3000 \mathrm{rpm}$ is reflected in the corrected pressure data as shown in Fig. 19. Because the linear model is being evaluated, the corrected pressure data is normalized by propeller-wash velocity. By comparing yaw moment coefficients computed from pressure and FT measurements, it can be concluded that the distributed sensing scheme is capable of accounting for asymmetries due to the nonlinear aerodynamic effects encountered at low propeller rotation rates. Such characterization was previously shown to not be possible using a system that relied only on a single prop-wash probe measurement and linear coefficient.

From the previous comparisons, the distributed sensing scheme is shown to correctly measure both pitch and yaw moments when in a steady hover once the calibration is applied. Through embedded pressure measurements over the tail surfaces, the pressure-based sensing scheme is also capable of accounting for the nonlinear aerodynamic effects encountered in test cases with the propeller driven at $3000 \mathrm{rpm}$.

\section{Alpha25 and 5kCruise Cases}

The calibration was applied to data from the Alpha 25 and $5 \mathrm{kCruise}$ cases then compared to FT measurements. The corrected slopes of the pressure-based measurements for pitch and yaw are observed to accurately match FT data slopes. Although the data point locations agree to within a standard deviation, a distinct offset is also apparent. The results are plotted in Figs. 20 and 21. Natural units are used in these plots because an appropriate choice for reference air velocity has not been proposed.

As previously described, these offsets are due to moment contributions from the wing and fuselage in a nonzero freestream. This can be characterized through either a direct sensing scheme or through an experimentally derived offset coefficient model. This augmentation is described in Sec. VI.

The combined freestream and propeller-wash test cases demonstrate the ability of the pressure-based sensing scheme to account for varying flow regimes. The control authority available in a forward-flight situation is far greater than those induced by propeller 

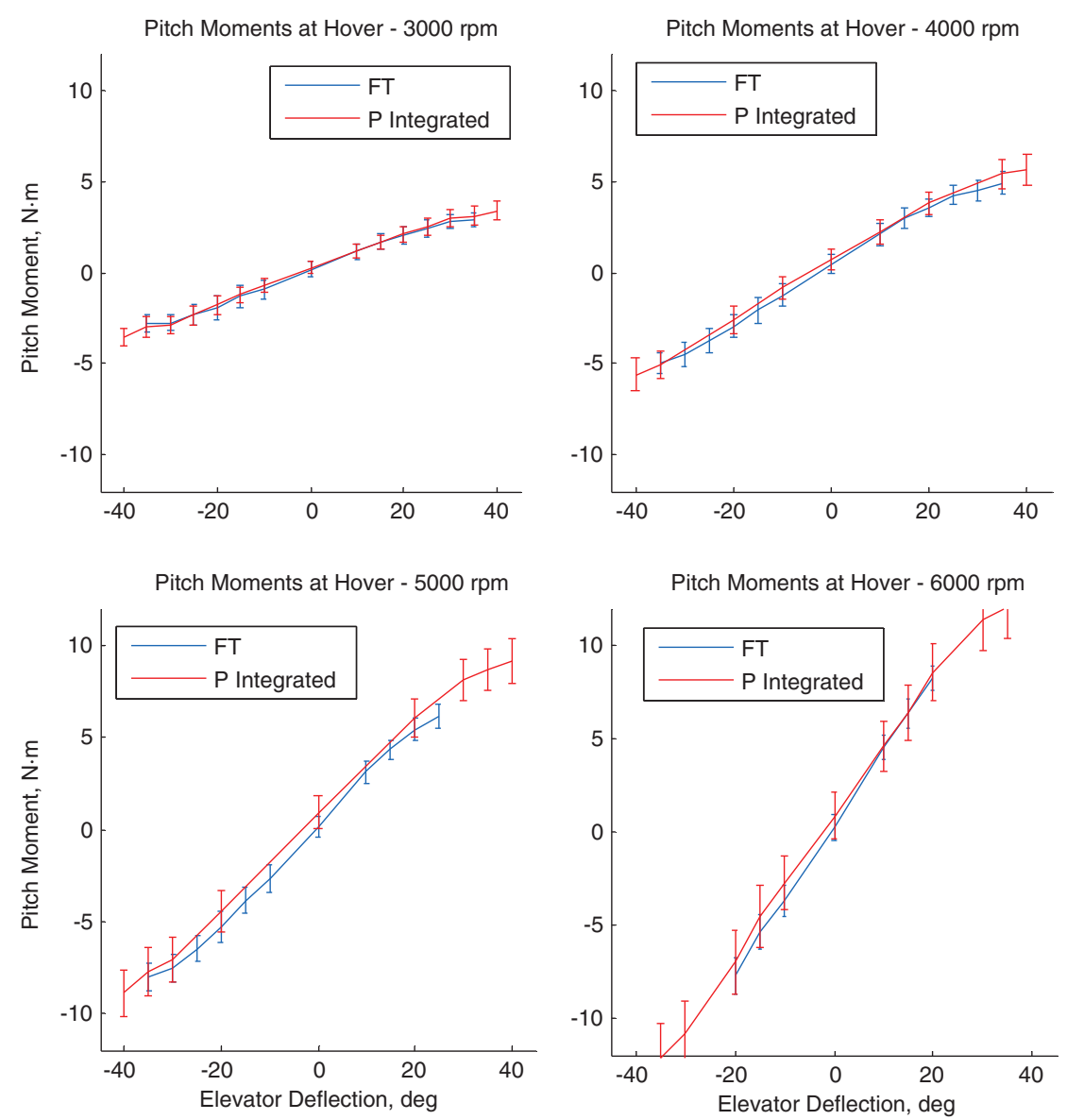

Fig. 17 Comparison between corrected pressure-based and FT measurements of hover pitch moments.

flow alone, as can be noted through much steeper slopes relating control surface deflection and resulting moments. Despite the significant change in flow conditions, the embedded pressure system
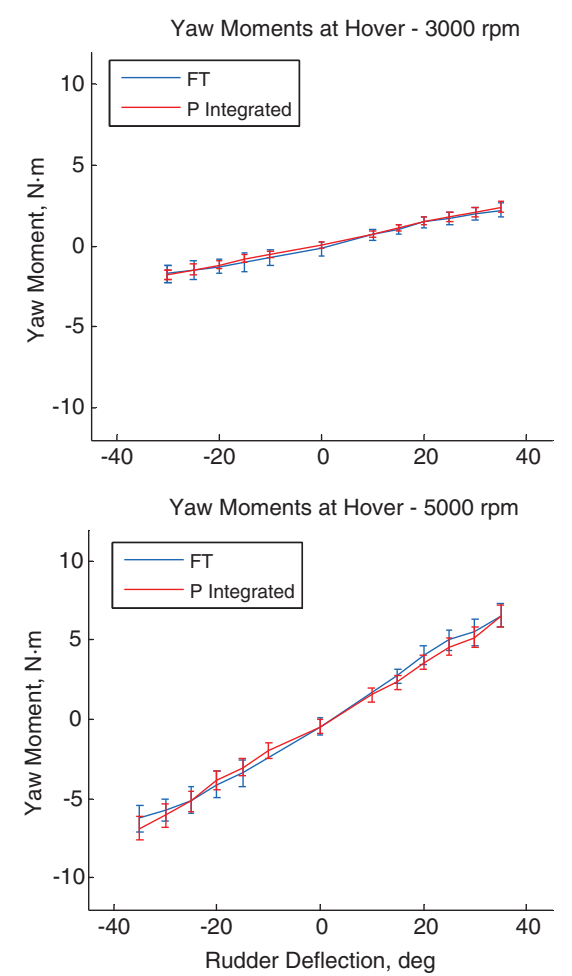

reports results that are also valid in forward flight. Based on these results, the calibration factors formulated at a low-turbulence cruise training data set is observed to be valid at all other test cases.
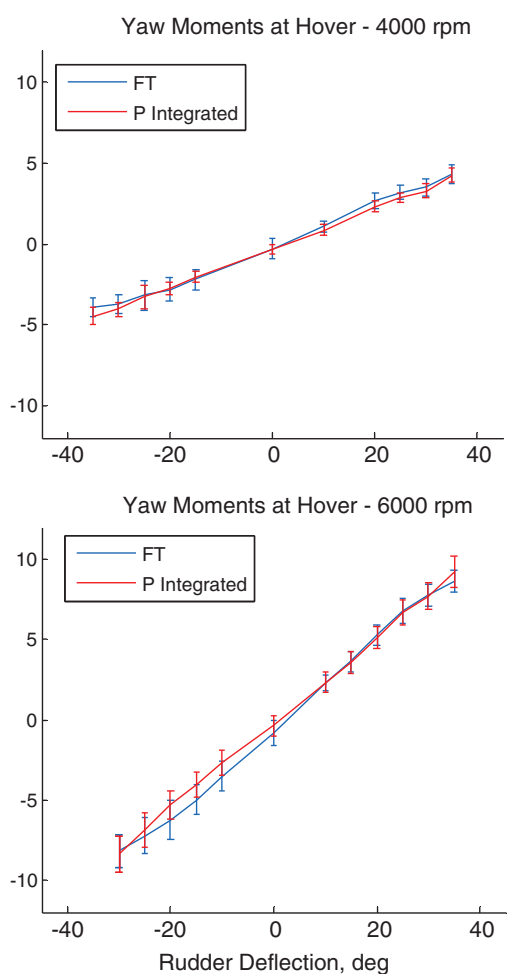

Fig. 18 Comparison between corrected pressure-based and FT yaw moment measurements for hover. 


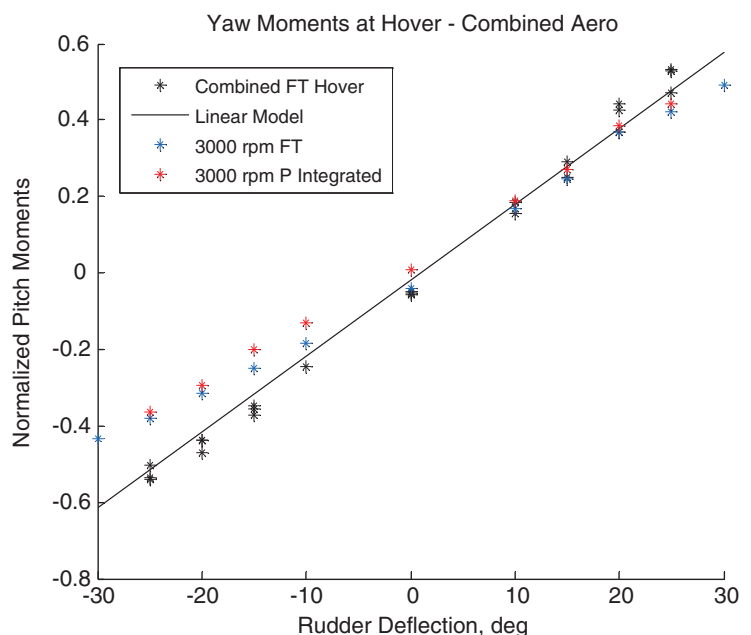

Fig. 19 Comparison of FT and pressure-based coefficients at $3000 \mathrm{rpm}$.

Therefore, it can be concluded that, with the distributed pressure instrumentation and a reference case, direct measurements of the aerodynamic moments generated by the tail surfaces can be taken.

\section{Conclusions}

This paper has proposed a reformulation of the steady-flight equations for fixed-wing aircraft operating at quasi-steady highangle-of-attack and hover conditions that includes prop-wash over the tail surfaces. The classical form of the equations for aerodynamic pitch moment $M$ and yaw moment $N$ do not allow for different flow velocities across the airframe to be considered. By adding a single propeller-wash velocity measurement, the effect of accelerated flow over the tail section can be incorporated as shown in the pitch and yaw equations in Eqs. (13) and (16), respectively.

The addition of a distributed pressure sensing scheme across the tail surfaces allows measurements of in-flight moments due to the elevator and rudder. At the cost of a more complicated instrumentation package, the reformulation in Eqs. (32) and (33) integrates distributed pressure sensing to directly measure pitch and yaw moments provided by aircraft tail surfaces.

Examples of a single-probe and a distributed sensing system have been implemented in an off-the-shelf Funtana aerobatic airframe. A wind-tunnel test setup has been developed that enables two approaches to implementing aerodynamic sensing to be explored: a partial sensing scheme with an innovative prop-wash air data probe, and a direct sensing scheme using distributed pressure sensing across the tail surfaces. An experimental procedure was described that allows both these approaches to be evaluated, and wind-tunnel test
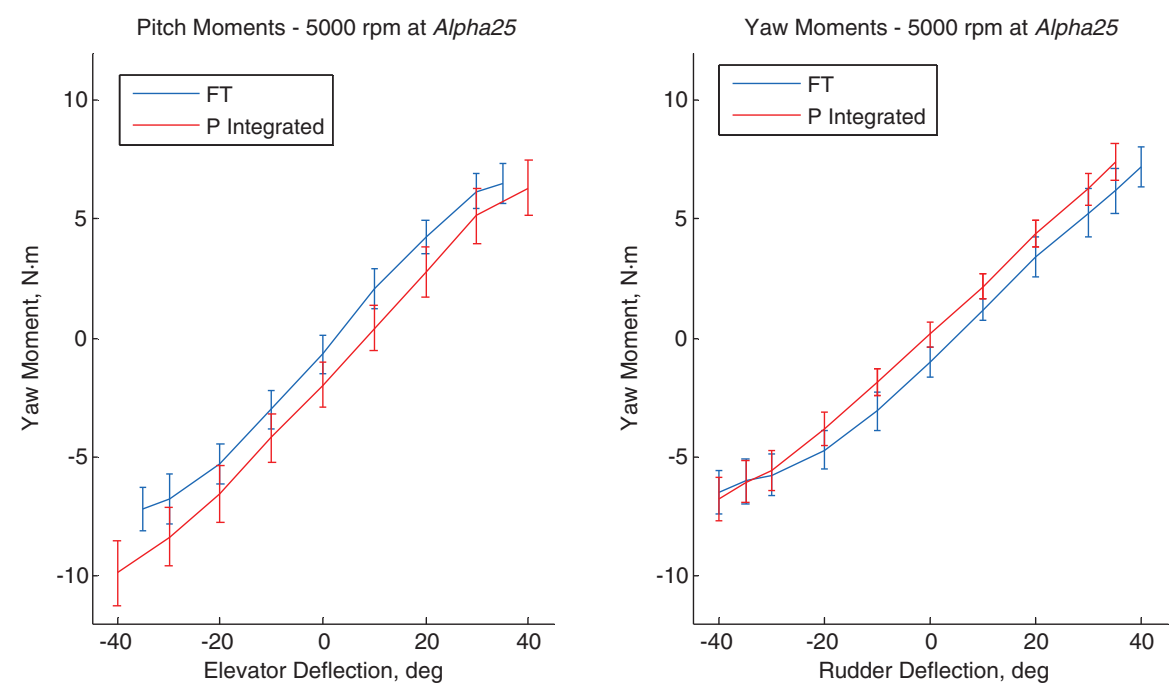

Fig. 20 Corrected pressure-based measurements at near-hover, Alpha25 case.
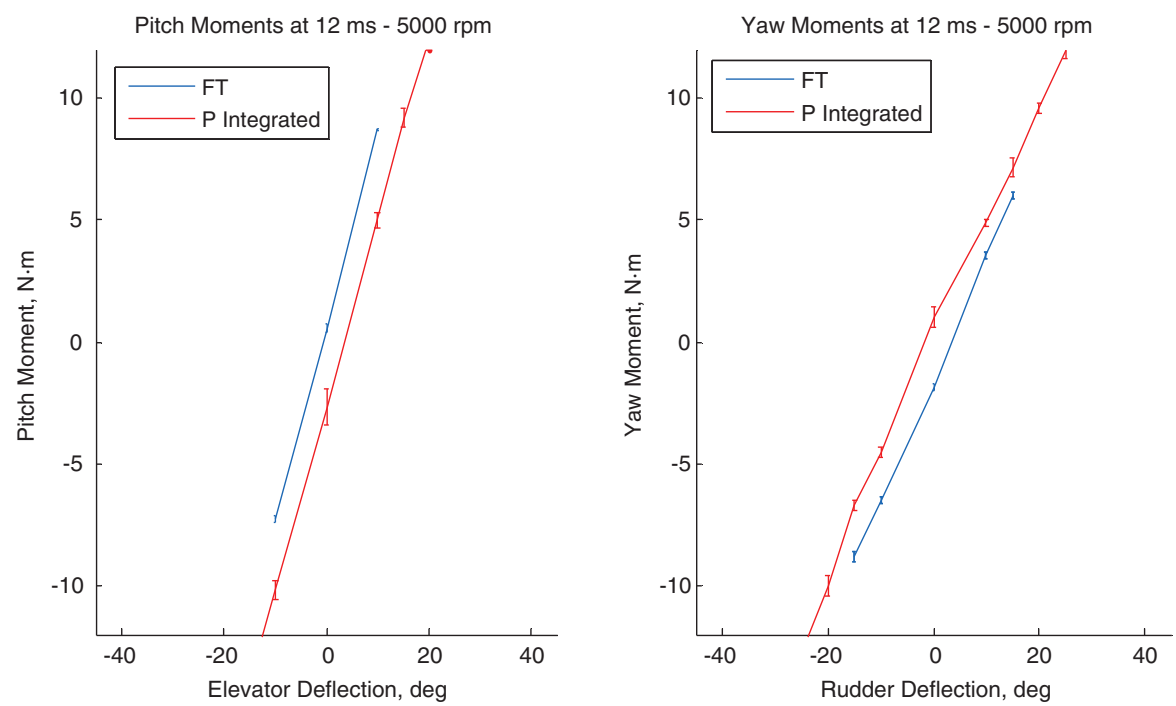

Fig. 21 Corrected pressure-based measurements at powered cruise. 
data from the experimental series have been presented. The following are key conclusions from this paper.

1) Wind-tunnel tests comparing integrated force-torque (FT), prop-wash probe, and direct pressure measurements show that both the prop-wash and the embedded pressure measurement schemes accurately characterize pitch and yaw moments during hover flight.

2) A simple moving average (SMA) filter designed by scaling the filter period to passing wakes shed by the propeller blades significantly reduces measurement uncertainty while maintaining a minimal response delay of the order of the propeller revolution time.

3) A partial aerodynamic sensing scheme using a novel propwash velocity measurement has been designed, implemented, and evaluated. The coefficients of a linear aerodynamics model for hover have been determined. Using additional aerodynamic feedback, the steady-flight equations for pitch and yaw moments have been extended to steady hovering flight, showing good agreement with direct FT sensor data in all hover test cases.

4) A direct pressure measurement approach to aerodynamic sensing has been proposed and experimentally evaluated for estimating moments induced by the aircraft tail. A further reformulation of the steady-flight equations allows the integration of distributed sensing across the tail surfaces for in-flight measurements of pitch and yaw moments. Results show that the embedded pressure sensing system provides accurate moment feedback for fixed-wing UAS operating at quasi-steady conditions including cruise, high angle of attack, and hover.

The work presented in this paper is part of an ongoing effort to realize flight control enhanced with more comprehensive aerodynamic feedback than has been previously used to support small overpowered UAS flight outside the traditional fixed-wing envelope. Although the distributed sensing scheme is capable of measuring moments under a wide variety of flight conditions, the large number of pressure measurements required to support it makes it challenging to implement. The prop-wash probe sensing system requires no additional embedded pressure ports but requires a more careful calibration able to characterize flow angles as well as speeds at high-angle conditions. In ongoing work, a full set of airframe static and dynamic model coefficients are being obtained to provide a more complete model using the prop-wash probe and embedded pressure sensors on the tail plus high-angle air data system and pressure sensors embedded in the wings as a means for full-aircraft in-flight aerodynamic force and moment characterization.

\section{References}

[1] “Unmanned Aircraft Systems Roadmap 2005-2030," U.S. Office of the Secretary of Defense, Washington, D.C., 2005, https://www.fas.org/irp/ program/collect/uav_roadmap2005.pdf [retrieved $20 \mathrm{Aug}$. 2013].

[2] Johnson, E., Wu, A., Neidhoefer, J., Kannan, S., and Turbe, M., "FlightTest Results of Autonomous Airplane Transitions Between SteadyLevel and Hovering Flight," Journal of Guidance, Control, and Dynamics, Vol. 31, No. 2, March-April 2008, pp. 358-370. doi:10.2514/1.29261

-[3] Johnson, B., and Lind, R., "Characterizing Wing Rock with Variations in Size and Configuration of Vertical Tail," Journal of Aircraft, Vol. 47, No. 2, March-April 2010, pp. 567-577. doi:10.2514/1.45719

[4] Chambers, J., "High-Angle-of-Attack Aerodynamics: Lessons Learned," AIAA 4th Applied Aerodynamics Conference, AIAA Paper 1986-1774, June 1986.

[5] Chambers, J., and Grafton, S., "Aerodynamic Characteristics of Airplanes at High Angles of Attack," NASA TM-74097, 1977.

[6] Nguyen, L., "Simulator Study of Stall/Post-Stall Characteristics of a Fighter Airplane with Relaxed Longitudinal Static Stability," NASA TP-1536, Dec. 1979.

[7] Simon, J., "Dynamic Derivative Data for High Angle of Attack Simulation," AIAA Guidance Navigation, and Control Conference, AIAA Paper 1992-4355, Aug. 1992.

[8] Bauer, J., Clarke, R., and Burken, J., "Flight Test of the X-29A at High Angle of Attack: Flight Dynamics and Control," NASA TP-3537, 1995.

[9] Herwitz, S., Allmendinger, K., Slye, R., Dunagan, S., Lobitz, B., Johnson, L., and Brass, J., "Nighttime UAV Vineyard Mission: Challenges of See-and-Avoid in the NAS," AIAA 3rd "Unmanned
Unlimited" Conference, Workshop and Exhibit, AIAA Paper 20046417, Sept. 2004.

[10] Beard, R., Kingston, D., Quigley, M., Snyder, D., Christiansen, R., Johnson, W., McLain, T., and Goodrich, M., "Autonomous Vehicle Technologies for Small Fixed Wing UAVs," Journal of Aerospace Computing, Information, and Communication, Vol. 2, No. 1, Jan. 2005, pp. $92-108$.

doi:10.2514/1.8371

[11] Hirokawa, R., Kubo, D., Suzuki, S., Meguro, J., and Suzuki, T., “A Small UAV for Immediate Hazard Map Generation," AIAA Infotech@Aerospace 2007 Conference and Exhibit, AIAA Paper 2007-2725, May 2007.

[12] Hsiao, F., Ding, Y., Chuang, C., Lin, C., and Huang, Y., "The Design of a Small UAV System as a Testbed of Formation Flight," AIAA Infotech@Aerospace 2011, AIAA Paper 2011-1422, March 2011.

[13] Eubank, R., Atkins, E., and Macy, D., "Autonomous Guidance and Control of the Flying Fish Ocean Surveillance Platform," AIAA Infotech@Aerospace Conference, AIAA Paper 2009-2021, April 2009.

[14] Rasmussen, N., Morse, B., and Taylor, C., "A Fixed-Wing, Mini-UAV System for Aerial Search Operations," AIAA Guidance Navigation and Control Conference and Exhibit, AIAA Paper 2007-6819, Aug. 2007.

[15] Xie, P., Flores-Abad, A., Martinez, G., and Ma, O., "Development of a Small UAV with Autopilot Capability," AIAA Atmospheric Flight Mechanics Conference, AIAA Paper 2011-6449, Aug. 2011.

[16] Kaloust, J., "Enhanced Stall and Recovery Control System (ESARCS): A Nonlinear Model-Based Flight Control System For Uninhabited Airborne Vehicles (UAV)," AIAA 1st Technical Conference and Workshop on Unmanned Aerospace Vehicles, AIAA Paper 2002-3489, May 2002.

[17] Shields, M., and Mohseni, K., "Limitations of Using the Linearized Equations of Motion for MAV Control," AIAA Guidance Navigation and Control Conference, AIAA Paper 2011-6221, Aug. 2011.

- [18] Green, W., and Oh, P., "A MAV That Flies Like an Airplane and Hovers Like a Helicopter," Proceedings of the IEEE/ASME International Conference on Advanced Intelligent Mechatronics, IEEE Publ., Piscataway, NJ, July 2005, pp. 693-698.

[19] Frank, A., Valenti, M., Leveine, D., and How, J., "Hover Transitions and Level Flight Control Design for a Single-Propeller Indoor Airplane," AIAA Guidance Navigation and Control Conference and Exhibit, AIAA Paper 2007-6318, Aug. 2007.

[20] Cory, R., and Tedrake, R., "Experiments in Fixed-Wing UAV Perching," AIAA Guidance Navigation and Control Conference, AIAA Paper 2008-7256, Aug. 2008.

[21] Selig, M., "Modelling Propeller Aerodynamics and Slipstream Effects on Small UAVs in Realtime," AIAA Guidance, Navigation and Control Conference, AIAA Paper 2010-7938, Aug. 2010.

[22] Selig, M., "Modelling Full-Envelope Aerodynamics of Small UAVs in Realtime," AIAA Guidance, Navigation and Control Conference, AIAA Paper 2010-7635, Aug. 2010.

[23] Johnson, E., and Kannan, S., "Adaptive Flight Control for an Autonomous Unmanned Helicopter," AIAA Guidance, Navigation, and Control Conference, AIAA Paper 2002-4439, Aug. 2002.

[24] Yeo, D., Henderson, J., and Atkins, E., "An Aerodynamic Data System for Small Hovering Fixed-Wing UAS," AIAA Guidance, Navigation, and Control Conference, AIAA Paper 2009-5756, Aug. 2009.

[25] Yeo, D., Atkins, E., Bernal, L., and Shyy, W., "Aerodynamic Sensing for a Fixed Wing UAS Operating at High Angles of Attack," AIAA Atmospheric Flight Mechanics Conference, AIAA Paper 2012-4416, Aug. 2012.

[26] Patel, M., Sowle, Z., Corke, T., and He, C., "Autonomous Sensing and Control of Wing Stall Using a Smart Plasma Slat," 44th AIAA Aerospace Sciences Meeting, AIAA Paper 2006-1207, Jan. 2006.

[27] Bowles, P., and Corke, T., "Stall Detection on a Leading-Edge Plasma Actuated Pitching Airfoil Utilizing Onboard Measurement," 47th AIAA Aerospace Sciences Meeting, AIAA Paper 2009-0093, Jan. 2009.

[28] Cox, C., Gopalarathnam, A., and Hall, C., "Flight Test of Stable Automated Cruise Flap for an Adaptive Wing Aircraft," Journal of Aircraft, Vol. 47, No. 4, 2012, pp. 1178-1188. doi: $10.2514 / 1.46789$

[29] Barnwell, W., Chokani, N., and Hall, C., "UAV Flight Control Using Distributed Actuation and Sensing," M.S. Thesis, Aerospace Engineering Dept., North Carolina State Univ., Raleigh, NC, 2003.

[30] Lion, S., and Hall, C., "Control Authorities of a Distributed Actuation and Sensing Array on a Blended-Wing-Body Uninhabited Aerial Vehicle," M.S. Thesis, Aerospace Engineering Dept., North Carolina State Univ., Raleigh, NC, 2007.

[31] "Multidisciplinary University Research Initiative Grant FA9550-05-10411," U.S. Air Force Office of Scientific Research, Wright-Patterson AFB, OH, http://www.avocet.gatech.edu [retrieved 22 Aug. 2013].

[32] Morelli, E., and DeLoach, R., "Wind Tunnel Database Development Using Modern Experiment Design and Multivariate Orthogonal 
Functions," AIAA 41 st Aerospace Sciences Meeting, AIAA Paper 20030653, Jan. 2003.

[33] Guiler, R., and Huebsch, W., "Wind Tunnel Analysis of a Morphing Swept Wing Tailless Aircraft," AIAA Applied Aerodynamics Conference, AIAA Paper 2005-4981, June 2005.

[34] Marmier, P., and Wereley, N., "Morphing Wings of a Small Scale UAV Using Inflatable Actuators for Sweep Control," 44th AIAA Structure Structural Dynamics and Materials Conference, AIAA Paper 20031802, April 2003.

[35] Landman, D., Britcher, C., and Bennet, W., "A Power-On Full-Scale Wind Tunnel Test of a UAV," 17th Applied Aerodynamics Conference, AIAA Paper 1999-3166, 1999.

[36] Ol, M., Zeune, C., White, T., and Kudla, T., "Wind Tunnel Evaluation of Powered Static Aerodynamics of an Aerobatic UAV," AIAA 51st Aerospace Sciences Meeting, AIAA Paper 2013-0241, Jan. 2013.
[37] McClamroch, N., Steady Aircraft Flight and Performance, Princeton Univ. Press, Princeton, NJ, 2011, pp. 105-112, 182-190.

[38] Yeo, D., "Aerodynamic Sensing for Autonomous Unmanned Aircraft Systems," Ph.D. Dissertation, Aerospace Engineering Dept., Univ. of Michigan, Ann Arbor, MI, 2013.

[39] Mallipudi, S., Selig, M., and Long, L., "Use of a Four-Hole Cobra Pressure Probe to Determine the Unsteady Wake Characteristics of Rotating Objects," 24th AIAA Aerodynamic Measurement Technology and Ground Testing Conference, AIAA Paper 2004-2299, JuneJuly 2004.

[40] Glauert, H., The Elements of Aerofoil and Airscrew Theory, Cambridge Univ. Press, Cambridge, England, U.K., June 1983, p. 199. 
This article has been cited by:

1. Daniel B. Quinn, Yous van Halder, David Lentink. 2017. Adaptive control of turbulence intensity is accelerated by frugal flow sampling. Journal of The Royal Society Interface 14:136, 20170621. [Crossref] 